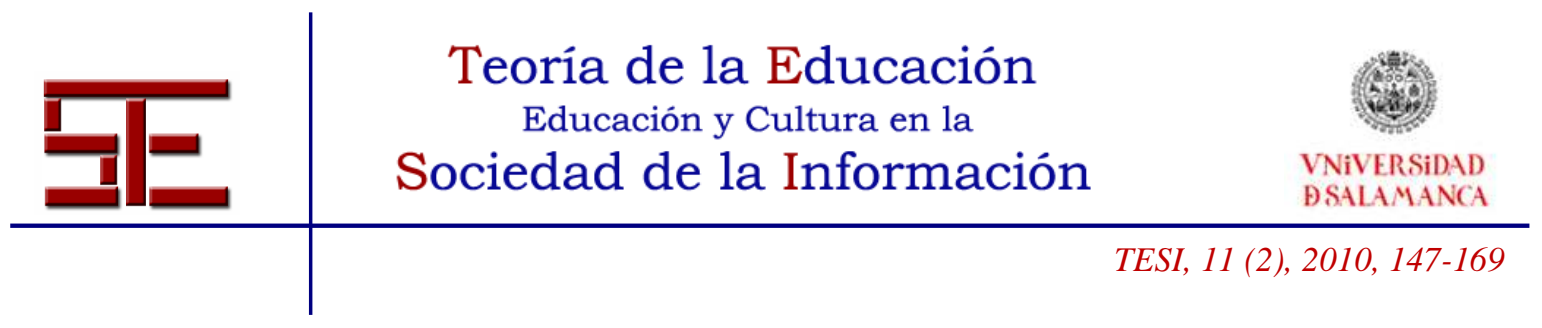

\title{
EL USO DE LAS SIMULACIONES EN EDUCACIÓN MÉDICA
}

Resumen:

En los últimos 20 años estamos asistiendo de forma progresiva al uso generalizado de las simulaciones en la formación de los médicos y de otros profesionales de las ciencias de la salud, en las diferentes etapas de su continuum educativo (grado, postgrado y formación continua). Tanto es así que ha surgido el concepto de la educación médica basada en las simulaciones, reconocida actualmente como una ayuda fundamental para asegurar el aprendizaje del estudiante y del médico y para mejorar la seguridad del paciente. En este artículo describiremos las razones por las que surge y se desarrolla esta nueva metodología, sus ventajas, los diferentes modelos y recursos disponibles en la actualidad y las características de los denominados centros de simulación o de habilidades clínicas que se han ido desarrollando en todo el mundo, en los cuales se lleva a cabo esta nueva modalidad de enseñanza médica. Así mismo trataremos brevemente la situación en nuestro país y finalmente nos referiremos a los principios de en los que se basa un desarrollo adecuado de la educación médica basada en las simulaciones.

Palabras clave: Simulación; Laboratorios de Habilidades; Centros de simulación; Educación médica basada en la simulación

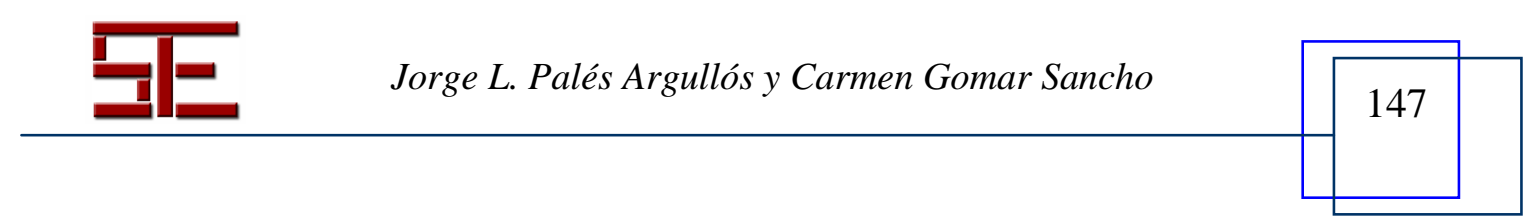




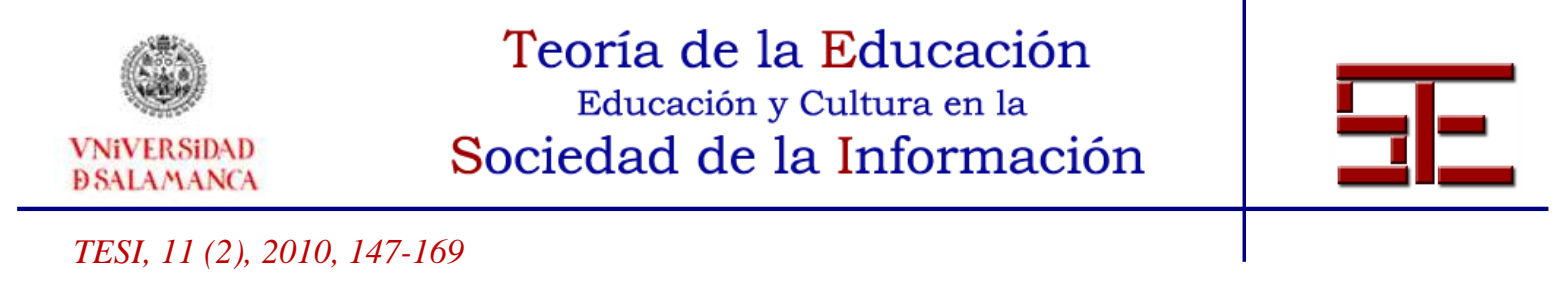

\title{
SIMULATION IN MEDICAL EDUCATION
}

\begin{abstract}
:
In last twenty years, we are seeing increasingly widespread use of simulations in the training of doctors and other professionals of health sciences at the different stages of their educational continuum (undergraduate, postgraduate and continuing medical education). So much so that the concept of simulations-based medical education has emerged and it is now recognized as a vital tool to ensure the learning of medical students and doctors, and to improve patient safety. This article will describe the reasons for the introduction and development of this new methodology, its advantages and the different models and currently available resources. We will describe also the characteristics of so-called simulation centres or clinical skills laboratories that have been developed worldwide, where the simulation-based medical education take place. Moreover, we will refer briefly to the situation in our country and finally to the principles of a good development of the simulation-based medical education.
\end{abstract}

Key words: Simulation; Skills laboratories; Simulation centres; Simulation based medical education

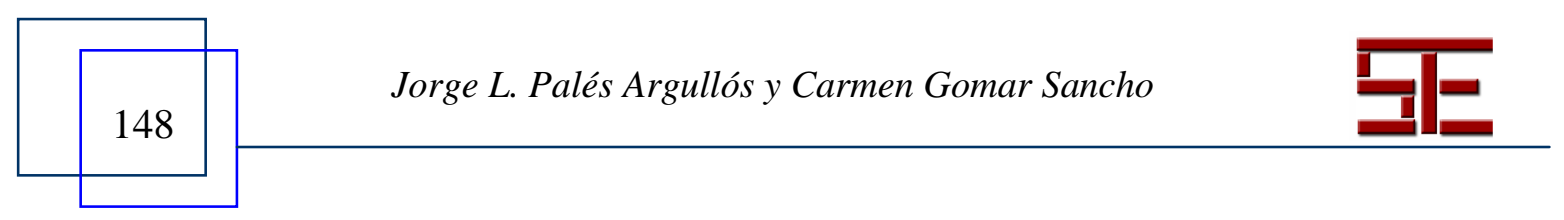




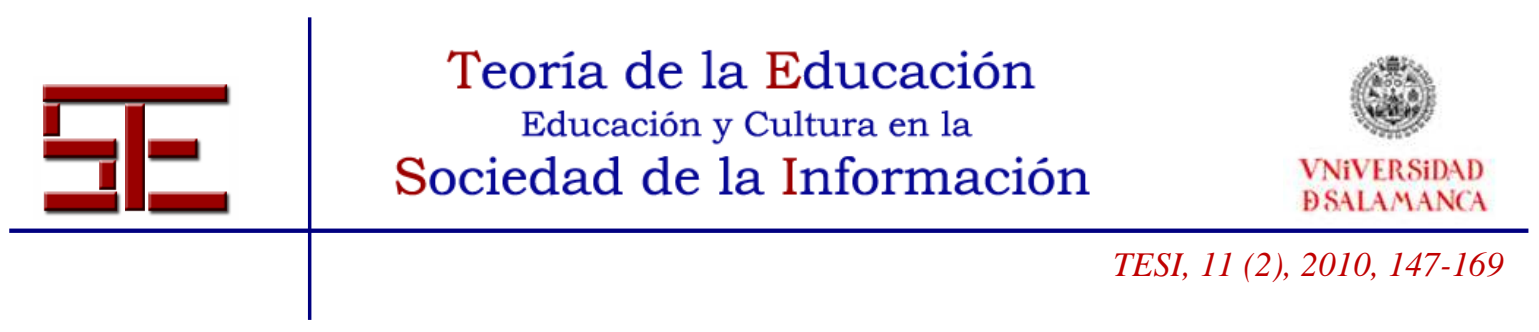

\title{
EL USO DE LAS SIMULACIONES EN EDUCACIÓN MÉDICA
}

Fecha de recepción: 15/12/2009; fecha de aceptación: 07/05/2010; fecha de publicación: 05/07/2010

\author{
Jorge L. Palés Argullós \\ jpales@ub.edu \\ Universidad de Barcelona \\ Carmen Gomar Sancho \\ cgomars@ub.edu \\ Universidad de Barcelona
}

\section{1.- INTRODUCCIÓN}

En los últimos 20 años, la utilización de las simulaciones en la educación médica se ha extendido de forma progresiva en todo el mundo como una forma de mejorar la formación de los profesionales de la salud en todas las etapas de su continuum educativo y como una forma de favorecer la seguridad de los pacientes y de evitar los errores médicos.

Las simulaciones son instrumentos educativos que se utilizan en el contexto de la denominada educación médica basada en las simulaciones que en sentido amplio podríamos definir como cualquier actividad docente que utilice la ayuda de simuladores con el fin de estimular y favorecer el aprendizaje simulando en lo posible un escenario clínico más o menos complejo. (Ziv, A. 2009). En este artículo vamos a describir como aparece y se desarrolla la educación médica basada en las simulaciones, sus ventajas, los recursos de los que disponemos en la actualidad para realizarla, los laboratorios de habilidades o centros de simulación, contextos en los cuales se desarrollan dichas actividades la situación actual en nuestro país y los principios básicos para realizar una buena educación médica basada en simulaciones.

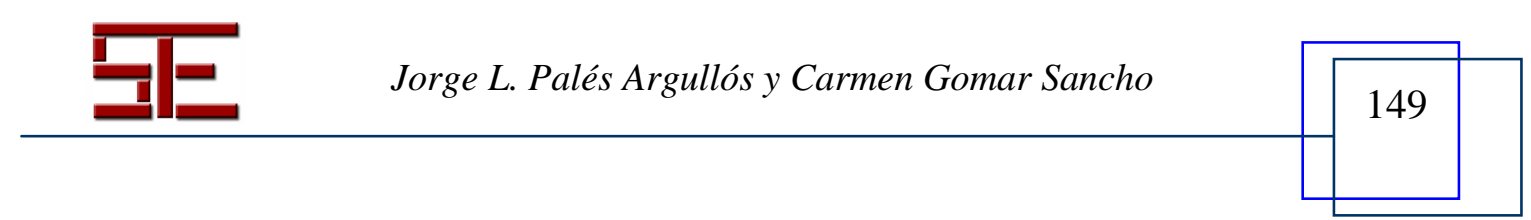




\section{Teoría de la Educación
Educación y Cultura en la
$\begin{aligned} & \text { VNiVERSIDAD } \\ & \text { DSALAMANCA }\end{aligned}$}

\section{2.- APARICIÓN Y DESARROLLO DE LA EDUCACIÓN MÉDICA BASADA EN SIMULACIONES. SUS CAUSAS.}

El uso de las simulaciones en diferentes contextos no es nuevo. De hecho las simulaciones vienen utilizándose desde hace tiempo en diversos campos. Podríamos citar como ejemplos, las plantas de energía nuclear que han tenido desde su inicio programas de seguridad basados en la simulación y en las que el conocimiento del reactor nuclear y el comportamiento ante una crisis nuclear se "ensaya" por simulación de forma regular o la industria aeronáutica donde se utilizan también simuladores en la formación de los pilotos de aviación. De hecho el primer simulador de vuelo aparece en 1929 introducido por el ingeniero estadounidense Edwin A. Link, pionero de la aviación que comercializó el denominado "Blue Box" o "Link Trainer" (Link EA, 1930). A partir de la segunda guerra mundial el desarrollo de simuladores para pilotos de aviación crece de forma exponencial y en la actualidad el $40 \%$ del tiempo de entrenamiento de pilotos de F16 se realiza en base al uso de simulaciones y el entrenamiento en el pilotaje de modelos nuevos de aeronave se hace exclusivamente por simulación. En el uso de las simulaciones en estos dos contextos subyace siempre el mismo principio: garantizar la seguridad y la prevención de errores críticos.

El caso de la educación médica no es diferente. En este sentido debemos referirnos al informe del Institute of Medicine de Estados Unidos de 1999 que con el título "Err is human”, (Kohn L.T. et al, 2000), estimaba en cerca de 100.000 anuales las muertes ocurridas en hospitales de aquel país como consecuencia de errores médicos, a parte del gasto económico generado por los daños a los pacientes. Se planteaba ya entonces la necesidad de intentar evitar estos errores médicos mediante una mejora de la formación de los profesionales.

Pero además es indispensable garantizar la seguridad y la intimidad de los pacientes durante el proceso de aprendizaje de dichos profesionales, lo cual se ha convertido en una exigencia ética. Esta exigencia ética puede entrar en conflicto con el aprendizaje realizado en pacientes en cualquier momento del proceso educativo de los profesionales. Como dice A. Ziv, "el uso de las simulaciones puede por un lado hacer más adecuada la formación de los profesionales y a la vez contribuir a minimizar el referido conflicto ético". (Ziv, A. et al, 2003).

Partiendo de esta exigencia de garantizar la seguridad del paciente que tiene sus raíces más antiguas en el aforismo o regla de oro de la medicina "Primum non nocere", en los últimos tiempos se han dado una serie de factores que han impulsado todavía más el uso de las simulaciones en educación médica y a los que nos referiremos a continuación. De

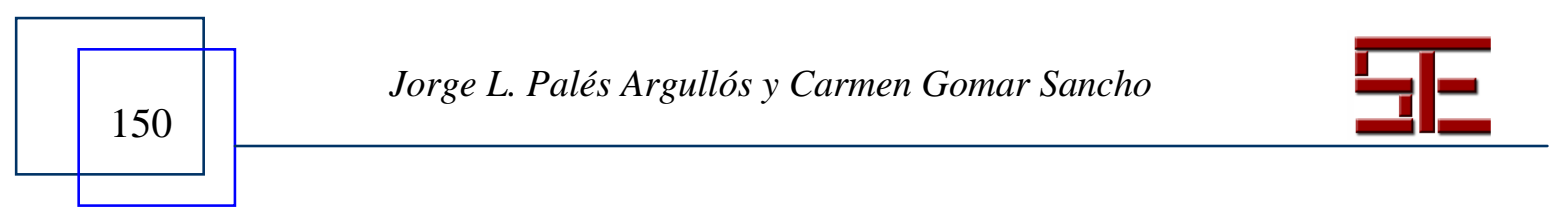




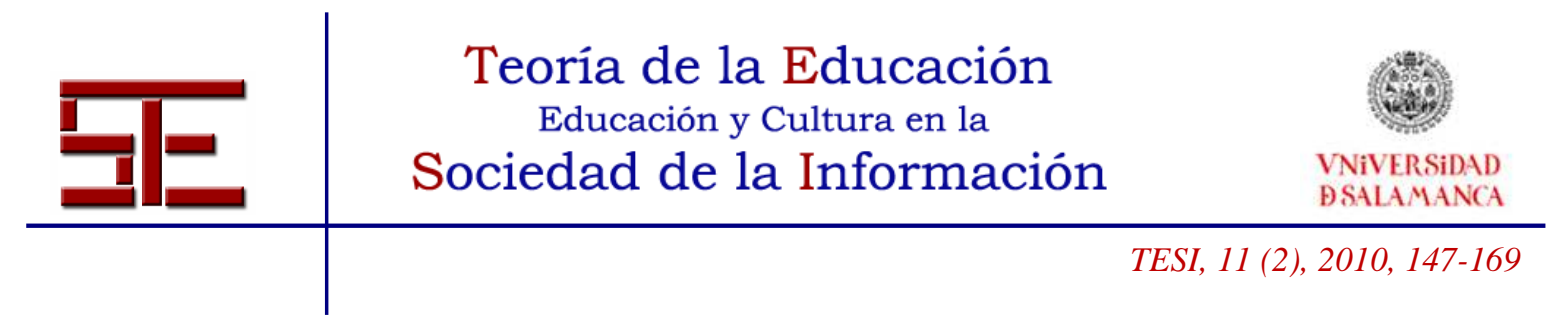

acuerdo con varios autores, (Ziv, A. y Berkenstad, H. 2008; Vázquez-Mata y Guillamet LLoveras, 2009, Mazarro, Palés y Gomar (2009), podríamos citar los siguientes:

1. Los programas para la seguridad y los derechos del paciente promovidas, entre otros, por la OMS.

2. Las demandas de responsabilidad médico legal que dificultan el modelo tradicional de aprender sobre los pacientes.

3. La restricción para la educación médica que ha supuesto la disminución de las horas de trabajo de los profesionales sanitarios en formación regulada en los países occidentales que disminuye el tiempo de exposición a los pacientes y obliga a buscar alternativas para garantizar una exposición clínica rica y estructurada.

4. Los cambios en el modelo asistencial sanitario que imposibilita que un paciente ingresado pueda ser sometido de forma repetida a exploraciones y procedimientos con objeto de entrenar a nuestros estudiantes, ya que esto supone molestias para los pacientes, posibles peligros para su seguridad al ser realizados por manos inexpertas y enlentecimiento de los procesos.

5. La presión de la actividad asistencial de los médicos que hace muy difícil una atención y supervisión adecuada de la actuación de los estudiantes por parte de los profesores.

6. Las evidencias de que las actuaciones de los profesionales en situaciones críticas poco frecuentes y la incoordinación de las actuaciones de los equipos asistenciales ante ellas solo puede adquirirse con simulación.

7. La importancia de asegurar la adquisición de las habilidades clínicas y de la capacidad del razonamiento clínico al mismo nivel que los conocimientos y en la necesidad de fomentar el aprendizaje autónomo de nuestros estudiantes.

8. El fomento por parte de organizaciones acreditadoras como el Educational Commission for Foreign Medical Graduates (ECFMG) y otras, de las evaluaciones del rendimiento de los profesionales de salud versus a las evaluaciones basadas en el conocimiento o cognitivas, para la obtención de la licencia para la práctica de la medicina o los procesos de reacreditación o recertificación.

9. La demanda de los movimientos de los derechos de los animales de evitar su uso para la enseñanza de los profesionales de salud

10. El impresionante desarrollo en los últimos tiempos de la investigación en el campo de la simulación, que está llevando a la creación de nuevos modelos de simulación cada vez mejores, más realistas y de más fidelidad para el aprendizaje y el entrenamiento y que ha determinado la aparición de grandes empresas que destinan inversiones importantes a la creación de dichos modelos.

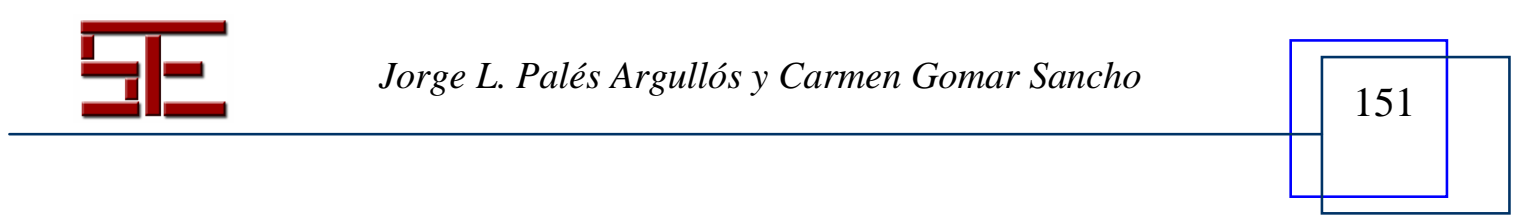




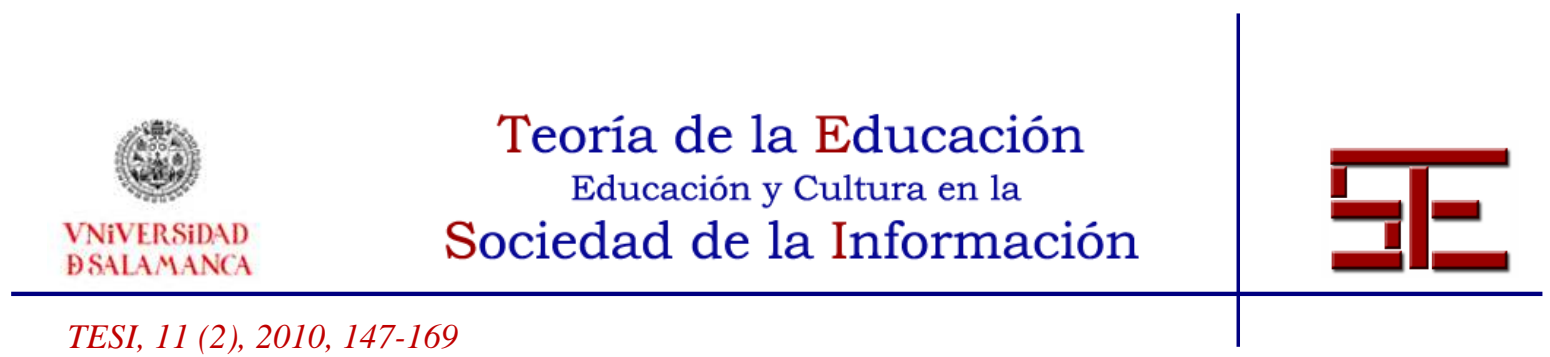

\section{3.- VENTAJAS EDUCATIVAS Y APLICACIONES DEL USO DE LAS SIMULACIONES EN EDUCACIÓN MÉDICA}

Además de solventar los problemas que plantea el cambio de modelo asistencial para la formación de los profesionales de la salud y de asegurar la intimidad del paciente e incrementar su seguridad, el uso de las simulaciones en educación médica comporta importantes ventajas desde el punto de vista educativo, y que convierten el entrenamiento basado en la simulación en la herramienta ideal para afrontar algunos de los nuevos retos de la educación médica.

Se ha podido demostrar que el uso de las simulaciones acorta el tiempo necesario para el aprendizaje de las habilidades, especialmente porque se puede repetir el entrenamiento tantas veces como sea necesario hasta adquirir las habilidades entrenadas y en un menor tiempo. Además las curvas de aprendizaje basadas en la simulación son mejores que las curvas basadas en el entrenamiento clásico. (Vázquez-Mata y Guillamet-LLoveras, 2009)

Por otra parte el entrenamiento basado en la simulación permite el error que se puede llevar hasta sus últimas consecuencias sin repercusiones reales. El alumno se puede enfrentar a situaciones desafiantes en un ambiente seguro donde el error está permitido y aprender de los errores sin dañar al paciente. De hecho se trata de una formación guiada por el error. Los errores son experiencias de aprendizaje y ofrecen grandes oportunidades de mejorar a través del aprendizaje de los mismos. (Ziv A. Berkenstad, H, 2008). La capacidad de aprender de los errores se multiplica al observar los alumnos los errores de sus compañeros.

El entrenamiento basado en la simulación permite corregir la falta de experiencia clínica y los fallos en la coordinación del equipo de profesionales. Es una formación orientada hacia el que aprende, teniendo en cuenta sus necesidades y su ritmo individual. La enseñanza basada en las simulaciones permite el aprendizaje de experiencias prácticas en diferentes tipos de entornos, desde los más simples a los más complejos, desde los más habituales a los poco comunes.

La enseñanza basada en las simulaciones permite que el alumno reciba feed-back en tiempo real de profesores y compañeros y reflexione sobre la acción por lo cual permite la evaluación de tipo formativo. Pero además al proveer un escenario o un entorno educativo estandarizado, reproducible y objetivo permite la evaluación con carácter sumativo. (Ziv A. 2007). Finalmente las habilidades adquiridas mediante la simulación son transferibles a la realidad.

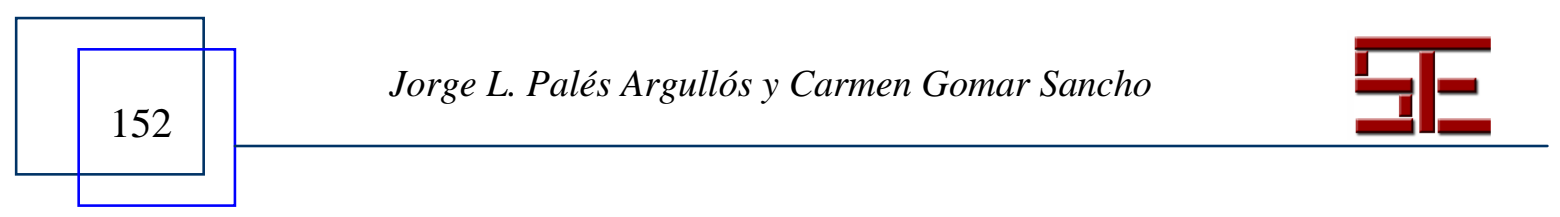




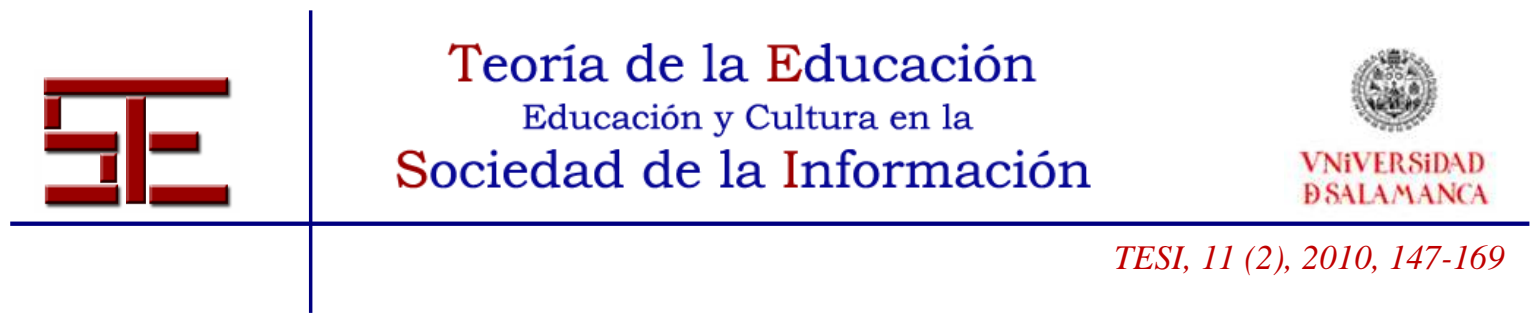

La educación médica basada en la simulación encuentra su aplicación en todas las etapas del continuum educativo de los profesionales de la salud, para adquirir experiencia en múltiples técnicas, reforzar la adquisición de habilidades deficitarias, y en la evaluación tanto de carácter formativo como para la licencia o la recertificación.

\section{4.- MODELOS Y RECURSOS DISPONIBLES}

Los primeros recursos disponibles en simulación surgen del campo de la Anestesiología. El primer maniquí médico se introduce en los años 60 para enseñar la reanimación cardiopulmonar básica con ventilación boca-boca. El "SimOne" se considera el primer simulador realista de anestesiología desarrollado en la universidad de California por Abrahamson y Denson, un ingeniero y un físico respectivamente, a finales de los años 1960, que se abandonó por su alto coste y por la falta de soporte de microinformática de la época y nunca se comercializó. (Denson JS Abrahamson S, 1969).

A mediados de los 80 investigadores de las universidades de Stanford y de Florida, trabajando independientemente empiezan a trabajar en la nueva generación de maniquíes de pacientes. Concretamente debemos citar el denominado Stanford CASE (Comprehensive Anaesthesia Simulation Environment) y que fue el primer recurso que se comercializó (Gaba DM, DeAnda A, 1988). En Europa, en los años 90, también de la mano de anestesistas se investiga en simulación, y se desarrollan simuladores con el mismo grado de sofisticación que los americanos. Podemos mencionar el simulador de anestesia de Leiden, (Chopra, V., Engbers, FHM., Geerts, MJ, 1994), el PAT Sim (Retterdal A. et al., 1996) y el simulador de anestesia SOPHUS (Christensen UJ. et al.1997.)

En los últimos 20 años se han ido introduciendo de forma incesante diferentes maniquíes bien para tareas o técnicas concretas o para simular el ambiente complejo de una situación clínica. El número de recursos para la simulación en la formación de los profesionales sanitarios se ha incrementado de forma impresionante disponiendo en la actualidad de modelos cada vez más reales y más fieles, con un rango amplio de niveles de complejidad y precio. (Tabla 1). Este proceso ha dado lugar a la creación de empresas dedicadas a la investigación en simulación y al diseño y comercialización de estos recursos que movilizan grandes cantidades de dinero. Repasemos brevemente los principales tipos de recursos que podemos agrupar en dos categorías: de baja tecnología y de alta tecnología. (Ziv, A. 2009, 2008) (Tabla 1)

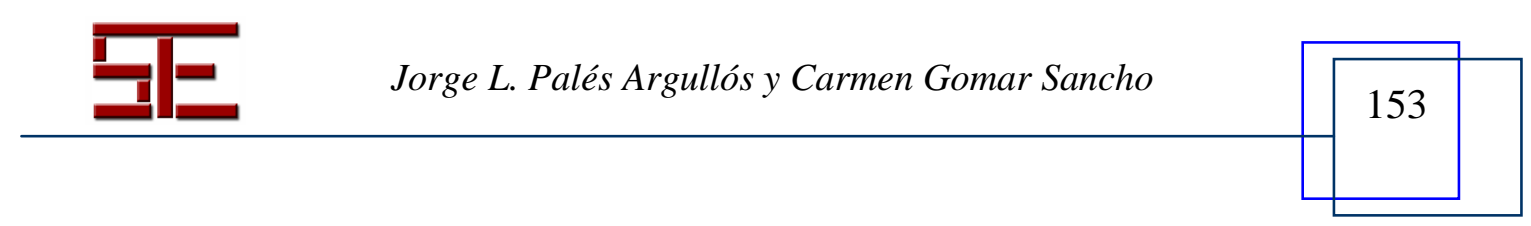




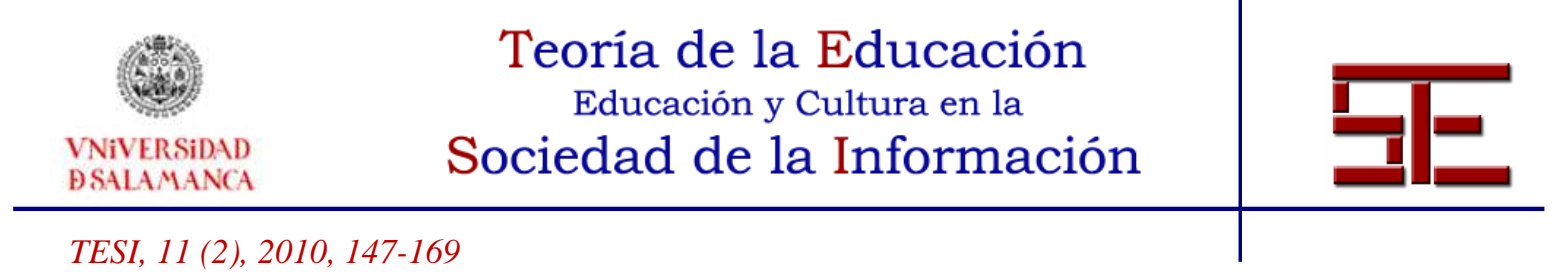

\section{1.- Simuladores de baja tecnología.}

Son simuladores sencillos mecánicos, de plástico o sintéticos (modelos o maniquíes) para practicar habilidades clínicas o procedimientos clínicos básicos como las punciones venosas, el tacto rectal, las suturas, punciones, las exploraciones ginecológicas, urológicas, oftalmológicas u otorrinolaringológicas, etc. Dentro de este grupo cabría incluir también los modelos sencillos para aprender técnicas de de intubación traqueal o como paso previo para los sistema de mayor complejidad de reanimación cardiopulmonar. También debemos considerar los modelos tridimensionales utilizados en la enseñanza de la anatomía. La mejor característica de todos estos modelos es su factibilidad ya que son muy accesibles tanto por su precio como por su baja complejidad de manejo. Dentro del apartado de simuladores de baja tecnología podemos incluir los modelos animales, los cadáveres humanos y los pacientes simulados o estandarizados. En las figuras 1 a 4 pueden apreciarse algunos de estos recursos utilizados por estudiantes de grado en su formación en habilidades y técnicas básicas.

La utilización de animales y cadáveres tiene su aplicación en la formación de grado especialmente en Anatomía y Farmacología y en la formación postgraduada en la cual se pueden entrenar procedimientos completos, situaciones quirúrgicas no previstas o nuevos instrumentos.

Los enfermos simulados o estandardizados o "pacientes actores" vienen utilizándose desde hace ya más de 30 años, siendo una práctica habitual en muchas facultades de medicina de todo el mundo. El uso de este tipo de recurso facilita el entrenamiento en habilidades de comunicación y la adquisición de competencias fundamentales como realizar la historia clínica y la exploración física. Aunque ninguna simulación es más real que un paciente real, los pacientes simulados se aproximan mucho a la realidad clínica y constituyen un instrumento de transición para enfrentarse a la clínica, siendo muy útiles para dar feed-back directo sobre las habilidades desarrolladas por los estudiantes.

Los pacientes simulados, al ser capaces de presentar el mismo cuadro de forma repetida, evitan las molestias excesivas a los enfermos reales. Los enfermos simulados alcanzan su máxima utilidad para adquirir aquellas habilidades de tipo genérico, no vinculadas a la especificidad del caso, pero no son útiles para la enseñanza de las habilidades de procedimientos técnicos. Si bien los costos de selección, entrenamiento y utilización de este tipo de "pacientes actores" son altos, son sin embargo costeeficientes en relación al coste de tiempo del profesorado necesario para alcanzar resultados similares. (Cantrell M, 2009)

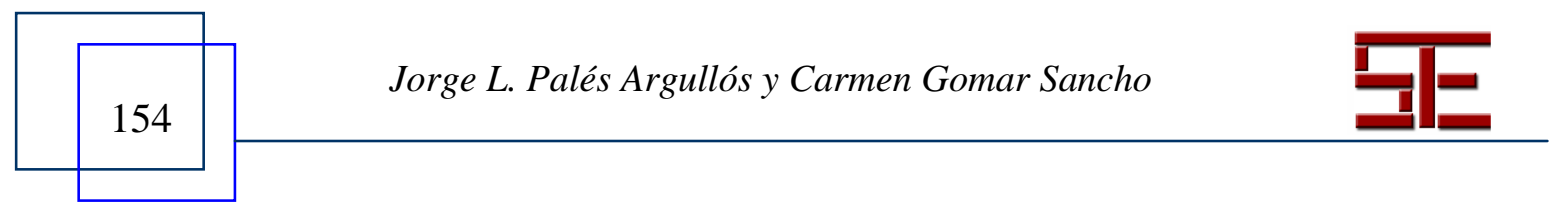




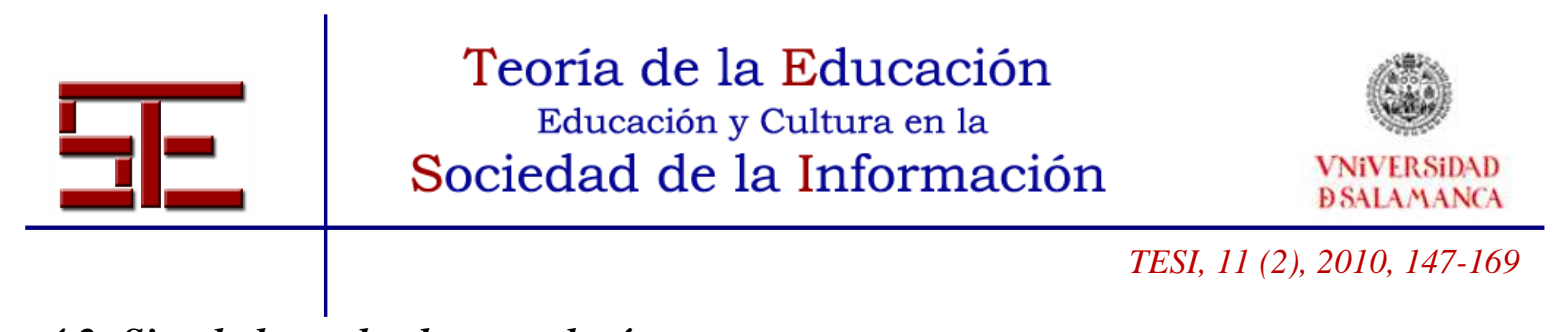

\section{2.-Simuladores de alta tecnología.}

En general son modelos basados en el uso de ordenadores, utilizando hardware y software con el fin de aumentar el realismo de la simulación. En este grupo debemos considerar:

a) Simulaciones por ordenador o mediante "pantalla" (screen simulation). Este tipo de modelos incluyen desde programas informáticos no interactivos hasta software interactivos complejos. Pueden utilizarse tanto en la enseñanza de ciencias básicas (anatomía, fisiología y farmacología) como de las clínicas. Facilitan el aprendizaje de los conocimientos, pero también el razonamiento clínico y la capacidad de decidir. El uso de estas simulaciones presenta ventajas educativas respecto al uso de pacientes reales en un considerable número de escenarios o situaciones clínicas. Todos los estudiantes pueden estudiar el mismo caso, es fácil dar feed-back en sus niveles de conocimientos y habilidades, pudiendo el estudiante cometer errores sin consecuencias y permiten disponer de diferentes patologías de las que no siempre se disponen en la realidad. Los programas pueden construirse con el elemento temporal incorporado de forma que es posible dar información al estudiante de las consecuencias de sus decisiones sobre el simulador. Las herramientas de autoevaluación que incorporan suelen ser buenas. Su generalización de uso depende de la disponibilidad de terminales u ordenadores, lo cual no representa un gran problema en nuestro entorno. El desarrollo del "software" tampoco supone un proceso excesivamente costoso. El aprendizaje con simulaciones por ordenador resuelve algunos de los problemas del aprendizaje con pacientes reales y compromete a los estudiantes tanto intelectualmente como emocionalmente en el aprendizaje. Se dispone ya de una gran cantidad de estos programas de simulación en todos los campos de la enseñanza de la medicina, muchos son ofrecidos de forma gratuita en Internet por sociedades científicas e instituciones docentes. Los comerciales suelen tener una buena relación costeefectividad.

b) Simuladores informáticos de gran fidelidad con recursos audiovisuales y táctiles e integrados para el entrenamiento de diversas tareas o procedimientos clínicos. Reproducen diversas tareas clínicas como auscultación cardíaca, cateterización cardíaca broncoscopia, colonoscopia, artrocentesis, endoscopia en diversos campos. En algunos modelos se asocian a metodologías de realidad virtual que ofrece diversas posibilidades en el entrenamiento médico de habilidades complejas como endoscopia, laparoscopia o navegación endovascular; en general, estas habilidades se dirigen más a la formación

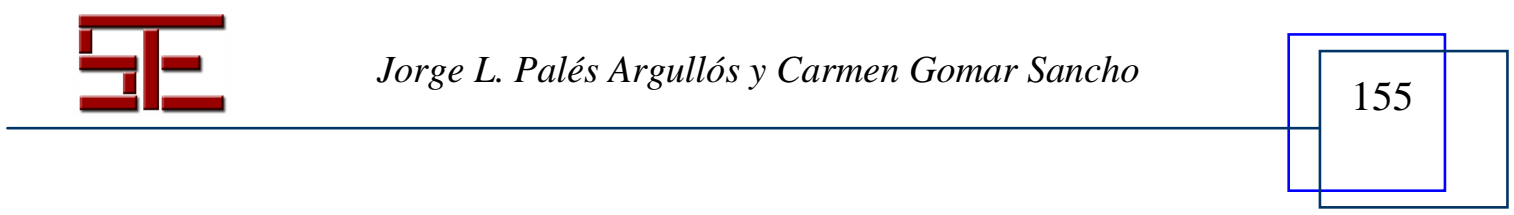




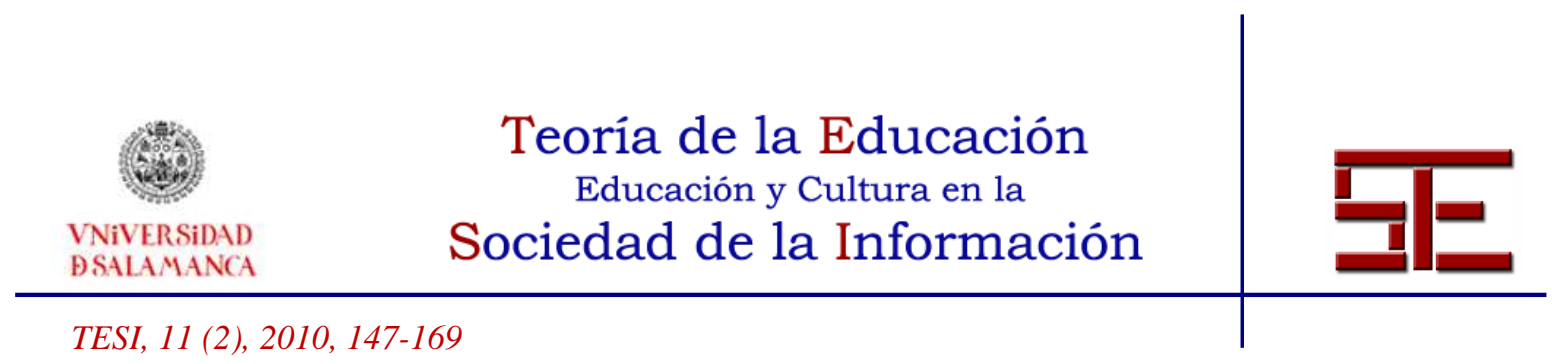

especializada que a la formación de grado. Existen múltiples modelos comercializados con un rango de precios muy amplio.

c) Simuladores de paciente completo interactivo realístico y de alta tecnología.

Son modelos fuertemente robotizados ligados a sistemas informáticos que aumentan enormemente las posibilidades de aprendizaje al permitir trabajar en múltiples situaciones fisiológicas y patológicas y manejar situaciones clínicas complejas en condiciones similares a la vida real. La simulación reproduce un cuerpo humano completo, con un software que dota al muñeco de todas las funciones cardíacas, vasculares y pulmonares. Esto permite diseñar síndromes/casos clínicos completos: el estudiante debe explorar al robot, llegar a una orientación clínica e iniciar un conjunto de habilidades básicas si la situación lo requiere. A partir de aquí, el nivel de complejidad puede elevarse. Suelen situarse en entornos decorados como las áreas asistenciales quirúrgicas o de reanimación y está demostrado de que ponen al alumno en un nivel de realismo muy bueno. Otra virtud de este tipo de entrenamiento que no se consigue con otro método docente, es enseñar a los distintos miembros de un equipo asistencial coordinación, lideraje y comunicación en actuaciones en situaciones críticas, de emergencia o en complicaciones vitales .La grabación y discusión (debriefing) de las actuaciones dentro de un entorno realista permite al alumno observar errores de comportamiento que no percibe por otro método. Dentro de esta categoría incluiríamos modelos que simulan el paciente adulto como METI-HPS, SimMan, para simulación obstétrica como NOELLE, pediátrica como PediaSim o BabySim.

A pesar de todas estas posibilidades, algunas de ellas muy complejas, debemos tener en cuenta que muchas veces no se siempre se requieren modelos demasiados complejos para el entrenamiento de determinadas habilidades. La simulación es una metodología docente, el simulador, sea de la complejidad que sea, un mero instrumento. Para cada objetivo docente hay un modelo de simulador suficiente y apropiado.

El mérito de un simulador no es su complejidad sino su utilidad y la frecuencia y aceptación para su uso por parte de los profesores. Situar la enseñanza por simulación solo en la alta tecnología supone abortar cualquier cambio cultural amplio en el profesorado y las instituciones. Ejemplos muy simples de simulaciones que cumplen los objetivos buscados son practicar las punciones intramusculares en una berenjena o una naranja, practicar suturas sobre tela, muslos de pollo o "patas de cerdo"o practicar la historia clínica o la exploración física normal entre los propios alumnos. Por otra parte la complejidad del recurso utilizado va en función del nivel en el que se realice la docencia, grado, postgrado o formación especializada. (Figura 5).

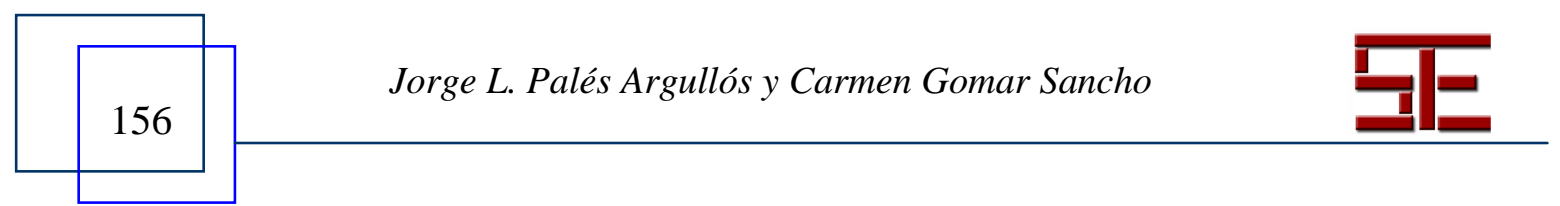




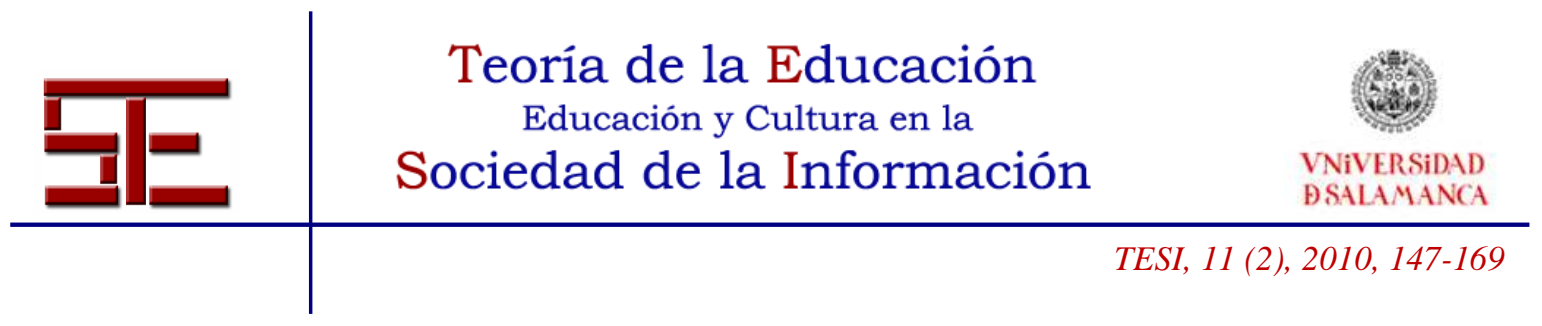

Si bien hoy en día disponemos de simuladores actuales de gran calidad, debemos ser conscientes de que en ningún caso, ningún simulador permite por si solo una enseñanza completa debiendo reconocer que es siempre parcial. La combinación de varios métodos de simulación y sobre todo la capacidad del profesor para aproximarlos a la realidad y conectarlos con la práctica clínica son las claves para obtener el máximo provecho. También hemos de ser conscientes que un simulador por muy complejo y perfecto que sea, nunca podrá compararse totalmente con la realidad, por lo cual hemos de ser siempre muy conscientes de sus limitaciones y que nunca suplirá totalmente el contacto con el paciente real, sino que lo ha de anteceder

\section{5.- LOS GRANDES CENTROS DE SIMULACIÓN Y LOS LABORATORIOS DE HABILIDADES CLÍNICAS. CARACTERÍSTICAS Y CONDICIONAMIENTOS PARA SU FUNCIONAMIENTO.}

La implantación de este nuevo paradigma de la educación médica basada en la simulación ha comportado la aparición de unos nuevos entornos educativos donde se lleva a cabo esta actividad docente. Nos referimos a los denominados laboratorios de habilidades en su versión más sencilla o de los grandes centros de simulación en su mayor complejidad.

En los últimos 20 años se ha producido una enorme proliferación de dichos centros. En general, podemos definir dichos centros como entornos educativos donde los estudiantes y los profesionales médicos o sanitarios, aprenden, mediante el uso de las simulaciones diferentes tipos habilidades en un entorno no estresante, pero próximo a la realidad, independientemente de la disponibilidad de pacientes reales, y bajo la supervisión de profesores o de forma autónoma e independiente. Son entornos donde es posible repetir, tantas veces como se desee, la técnica o habilidad concreta, que permiten el error sin consecuencias, su análisis detallado y su corrección y el control de la complejidad de la situación del aprendizaje y donde el estudiante recibe de forma continua, feed-back sobre su actuación y puede ser evaluado de forma objetiva.

En función de los recursos, los objetivos de aprendizaje. el tipo de alumnos y las fases de la educación médica a los que vaya dirigido el proceso formativo (grado, postgrado, formación continuada) podemos considerar los llamados laboratorios de habilidades en el contexto de centros universitarios (facultades de medicina, escuelas de enfermería, etc.) y cuyo objetivo es el aprendizaje por parte de los estudiantes del grado de las habilidades básicas, o los centros de simulación complejos, de alta complejidad, multidisciplinares y dotados con alta tecnología para la formación postgraduada y la formación continua, vinculados a centros hospitalarios o independientes, públicos y

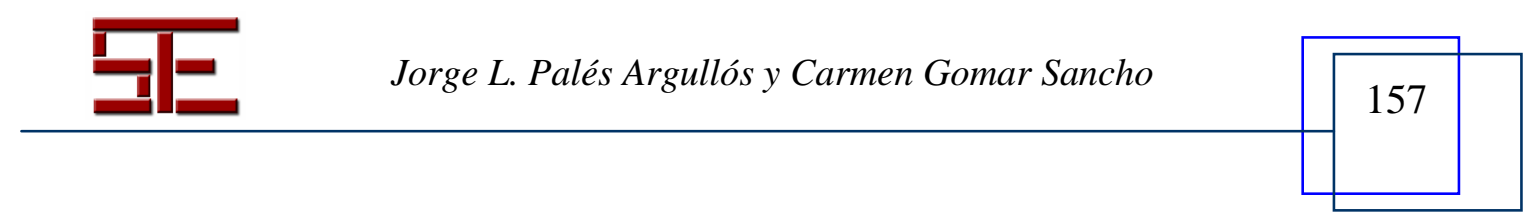




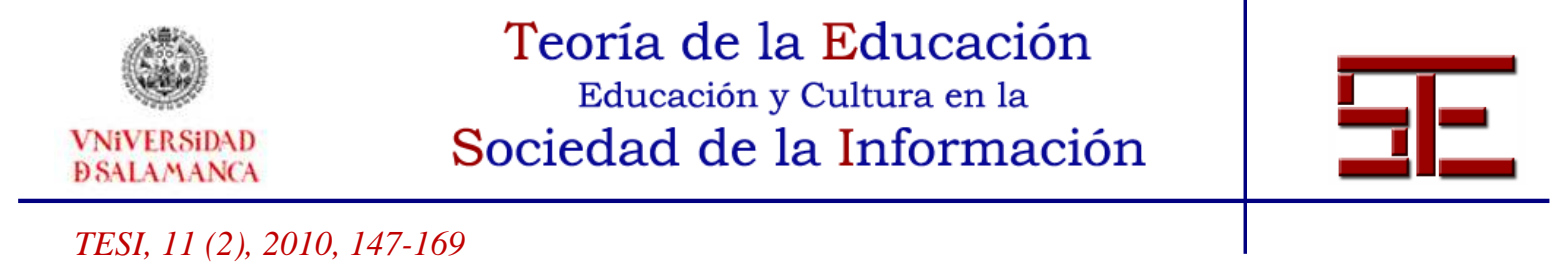

privados y que prestan servicios a diferentes instituciones y colectivos. Hemos de tener muy claro que el objetivo implementar este tipo de recursos no es hacerlo como un signo de modernidad y sofisticación de una institución, sino usarlo ampliamente, asiduamente y con la misma naturalidad que otros métodos docentes.

Los laboratorios de habilidades pueden consistir en simples espacios de mayor o menor tamaño, en forma de mini-laboratorios donde se enseñan algunas habilidades e incluso espacios de mayor o menor tamaño que remeden ámbitos clínicos, como un "box" de urgencias o un quirófano con todas sus prestaciones, y donde el abanico de las habilidades y procedimientos a practicar es muy amplio.

En el caso de los grandes centros de simulación o laboratorios más complejos deben tenerse en cuenta para su establecimiento diversos elementos claves como son el diseño físico del espacio que ha de ser lo más funcional posible, permitiendo una buena circulación del personal, y que disponga de zonas adecuadas para las diferentes actividades que se han de llevar a cabo en ellos (salas para la practicas de las diferentes habilidades y procedimientos, salas para el debriefing, aula, zona de descanso y zonas externas, etc. Estos espacios han de disponer de las características adecuadas como control de luz y de ventilación, aislamiento sonoro, sistemas audiovisuales, acceso a las tecnologías de la información y de la comunicación (TIC), etc. (Horley, R. 2008,; Vázquez, G, 2007)

Estos centros además suelen ser edificios interactivos, poseen laboratorios de entrenamiento, y salas talleres de análisis y retroalimentación; en estos espacios se encuentran los recursos de simulación que pueden dividirse en varios dos apartados: equipos para la simulación médica y equipos para la simulación en cirugía. Disponen de personal técnico, informático y docente. En general los equipos médicos corresponden a maniquís que simulan alguna parte del organismo humano, y permiten el entrenamiento en habilidades manuales básicas, o bien maniquís humano completos e interactivos que permiten reproducir la semiología básica cardiorrespiratoria, así como la función cardiovascular y pulmonar completas. Estos últimos maniquís, reproducen mediante un software, cuadros clínicos diversos, que el equipo médico y enfermero deberán de identificar y tratar. Los equipos de simulación quirúrgica suelen corresponder a equipos de realidad virtual y están dirigidos especialmente a las nuevas tecnologías quirúrgicas, soliendo combinarse zonas de entrenamiento en microcirugía, cirugía de mínima invasión y cirugía endoscópica y endovascular. Además, para cualquier área se escenifican situaciones críticas y emergentes para la actuación de los diversos profesionales que intervienen. Estos centros a su vez suelen apoyarse en un Campus Virtual, en los que se gestiona la parte administrativa de los cursos, a la vez que se da apoyo de eLearning, y como instrumento de comunicación y colaboración.

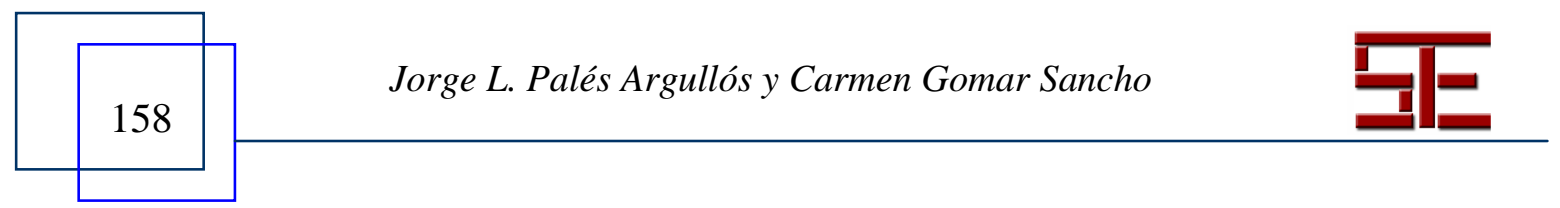




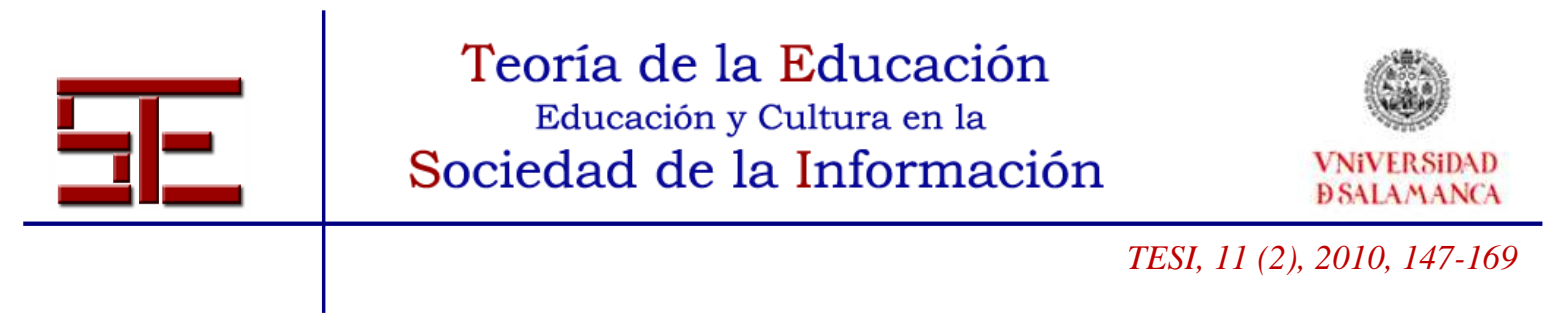

Paralelamente se necesitan espacios para almacenes, servicios administrativos y espacios para los especialistas no médicos que diseñan las estructuras docentes de los cursos.

Un ejemplo paradigmático de este tipo de centro es MSR, The Israel Center for Medical Simulation, líder internacional en el campo de la simulación médica, fundado en 2001 y dirigido por el Dr. Amitai Ziv, uno de los principales expertos mundiales en este campo. En la actualidad este centro permite la formación de más de 7000 profesionales de la salud cada año. Los detalles de este centro pueden consultarse en http://www.msr.org.il

Estos grandes centros dedicados exclusivamente la simulación requieren para su funcionamiento, desarrollar una serie de estrategias, que pueden esquematizarse en los siguientes puntos, (Vázquez, 2007):

a) Identificar una misión, valores y unos clientes apropiados a dicha misión.

b) Disponer de una financiación, global del centro y especifica por actividad, recurriendo a fuentes diversificadas, y asegurando su sostenibilidad geográfica, que asegure a la accesibilidad de las personas, y minimice todos los tipos de costes.

c) Disponer de plantillas, minimizando las plantillas estables, y buscando directores de cursos y tutores entre los profesionales clínicos en activo.

d) Diseño y producción de los cursos, mediante cadenas de producción diferenciadas

e) Realizar una gestión de la calidad en todas sus dimensiones, y centrada en procesos y resultados.

f) Innovar permanentemente mediante alianzas con los centros de conocimiento.

Pero también existen factores que pueden limitar su desarrollo. Entre ellos cabe citar: su rentabilidad y frecuencia de uso de estos centros, al estar a menudo alejados de los lugares de trabajo de los alumnos y profesores o los costes de los equipos de simulación, tanto robótica como virtual, sobre todo a nivel de los grandes centros y del personal muy cualificado que se precisa para que sean utilizados con frecuencia. (Vázquez, 2007)

El primer laboratorio de habilidades estructurado en una facultad de medicina Europa se establece formalmente en la Universidad de Maastricht en Holanda en 1974. Dicho laboratorio facilita entrenamiento en cuatro áreas bien definidas: habilidades de exploración física, habilidades terapéuticas, habilidades de laboratorio y habilidades de comunicación. A partir de aquí y en los últimos 25 años se produce una gran proliferación de estos laboratorios a nivel mundial, en el contexto de una facultad de medicina o de un hospital, o como un centro monográfico, siendo una constante en la

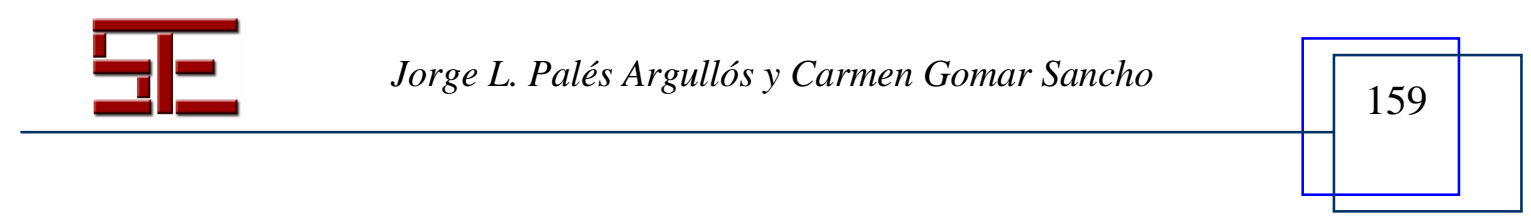




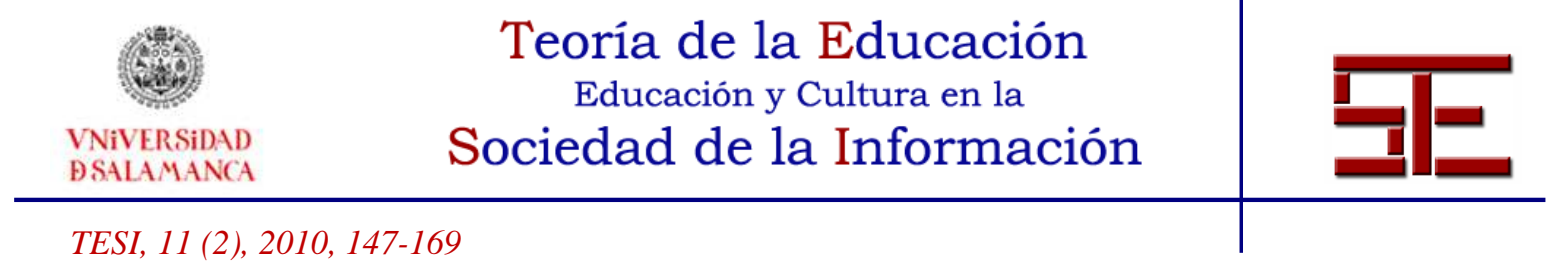

práctica totalidad de facultades de medicina de Estados Unidos, Canadá y Reino Unido, Israel y de otros países europeos avanzados.

Según la base de datos http://www.bmsc.co.uk/sim_database/centres_europe.htm a fecha de hoy el número de estos centros establecidos en cualquiera de sus formatos en todo el mundo es aproximadamente de más de 1430 centros, de los cuales aproximadamente 1000 se sitúan en EE.UU y Canadá, más de 200 en países europeos, incluyendo Israel, 23 en Sudamérica, 6 en países africanos, más de 160 en Asia y unos 30 en Australia. El lector interesado puede consultar la referida web donde podrá encontrar las referencias más importantes de todos y cada uno de estos centros, incluyendo su localización, sus datos de contacto, sus prestaciones y años de creación. A todos estos centros cabría añadir aquellos laboratorios de habilidades clínicas de dimensiones y complejidad más reducida que se han establecido en muchas facultades de medicina de todo el mundo, específicamente pensados para la formación de grado.

España en los últimos años se ha sumado también a este nuevo paradigma de la educación médica basada en las simulaciones, con la creación de tres grandes centros de simulación. Estas experiencias suponen distintos enfoques de la simulación, tanto en sus variantes con en su gestión. En primer lugar, por orden cronológico, el Centro de Cirugía de Mínima Invasión "Jesus Uson",(http://www.ccmijesususon.com/) ubicado en Cáceres desde 1995 y dedicado exclusivamente al entrenamiento en nuevas tecnológicas quirúrgicas en animales, y que representa el mayor complejo de entrenamiento quirúrgico de la Unión Europa. En segundo lugar el Centro de Entrenamiento en Situaciones Críticas Fundación Marcelino Botín, (http://www.cesc.com.es/), (Hospital Universitario Marqués de Valdecilla creado en Santander en 1997, localizado en el Hospital Marqués de Valdecilla de Cantabria, y que representa una alianza entre servicios hospitalarios e Instituciones extrahospitalarias, que ha permitido crear un servicio de simulación monotemático y es fácilmente asimilable en otros hospitales. Finalmente, la Fundación Iavante (http://www2.iavante.es/es/fundacion) creada en 2004 en Granada dependiente de la Junta de Andalucía dedicada a la transferencia del conocimiento y el entrenamiento de los profesionales de la sanidad en toda Andalucía, y que cubre tanto el entrenamiento del área médico / enfermero como la del área quirúrgica. Entre sus diversas sedes destaca el Centro Multifuncional Avanzado de Simulación e Innovación Tecnológica, localizado en Granada, Esta Fundación representa una apuesta de la Administración Pública Andaluza por la mejora permanente actualización de sus profesionales sanitarios.

A nivel de la enseñanza de grado, en los últimos años las facultades de medicina españolas, sobre todo en relación con la exigencia de adaptación al proceso de convergencia europea y la necesidad de formar a sus estudiantes en competencias han

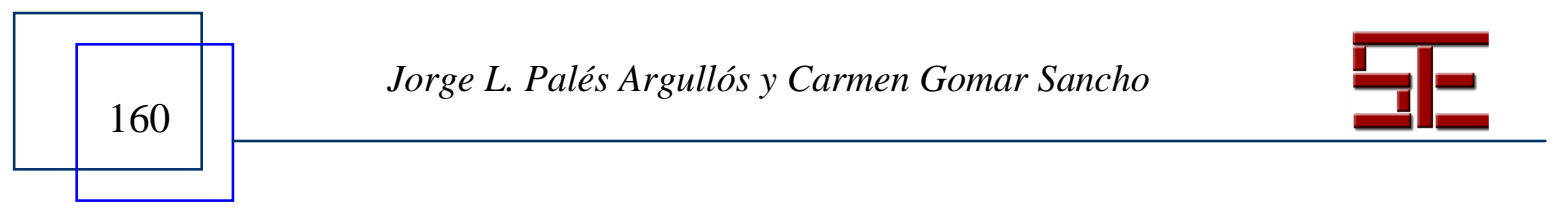




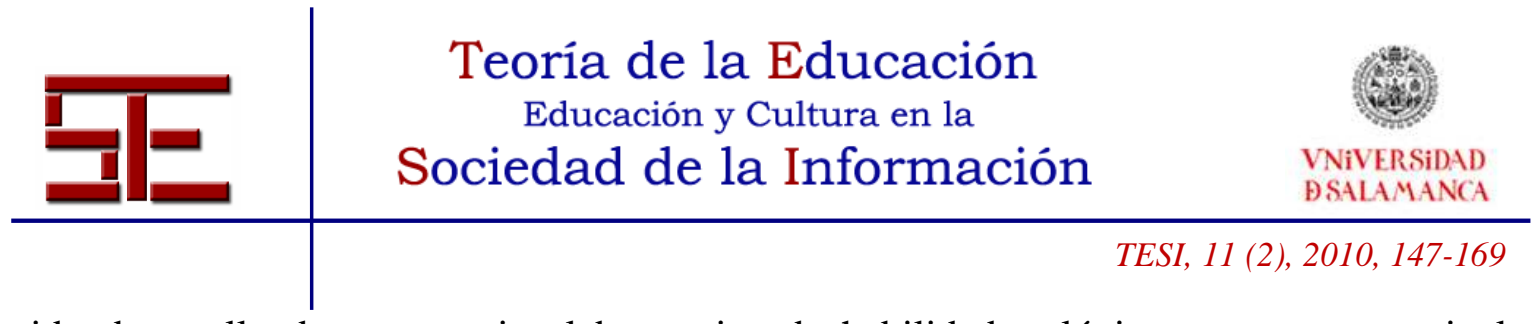

ido desarrollando sus propios laboratorios de habilidades, lógicamente con un nivel menor de complejidad que los grandes centros de simulación, y muchas de estas facultades han establecido estos servicios destinados a los alumnos del grado, e incorporando progresivamente las simulaciones a los curricula de medicina, aunque bien es verdad que todavía en algunos entornos no se acaba de percibir este tipo de enseñanza como una necesidad

Además, este tipo de actividades educativas no pueden afrontarse individualmente, al revés de lo que sucede con las clases magistrales y se requiere un rediseño de las estructuras de apoyo a la formación en facultades de medicina y hospitales. Los profesores y tutores necesitan también un entrenamiento específico en el manejo, diseño e implementación de este tipo de acciones formativas y el esfuerzo que se requiere para formarlos en esta nueva metodología, supera con creces el que se precisa para formarse en otras metodologías más tradicionales.

Actualmente nadie discute que un profesor debe utilizar con naturalidad y asiduidad medios audiovisuales o Internet para la enseñanza. De la misma manera debería ser indiscutible que un profesor de ciencias de la salud supiera utilizar con naturalidad la simulación.

\section{6.- LA SIMULACIÓN EN EDUCACIÓN MÉDICA COMO CAMPO CIENTÍFICO.}

El gran desarrollo de la educación médica basada en las simulaciones ha estimulado la creación de sociedades científicas sobre esta temática. A nivel internacional, debemos citar la Society for Simulation in Health Care (SSIH) http://www.ssih.org y la Society in Europe for Simulation Applied to Medicine (SESAM), http://www.sesam-web.org

La SSIH es la principal organización internacional que agrupa a profesionales procedentes de diferentes disciplinas y especialidades con el fin de intercambiar experiencias en simulación. La SSIH se creó en 2004 y tiene sus bases en diversas sociedades de anestesiología de los años 90. La SSIH organiza cada año en enero un congreso internacional sobre Simulación en salud y publica la revista Simulation in Healthcare, la única publicación multidisciplinaria y abarcando distintas especialidades, dedicada a la aplicación de las simulaciones en educación médica.

Por su parte la SESAM se funda en Copenhague en 1994 con el objetivo de promover el empleo de la simulación en medicina en Europa con fines docentes e investigadores y dar apoyo al uso de la simulación en medicina para la investigación y el entrenamiento. No está afiliada a ninguna especialidad médica o de otro tipo, y sus miembros

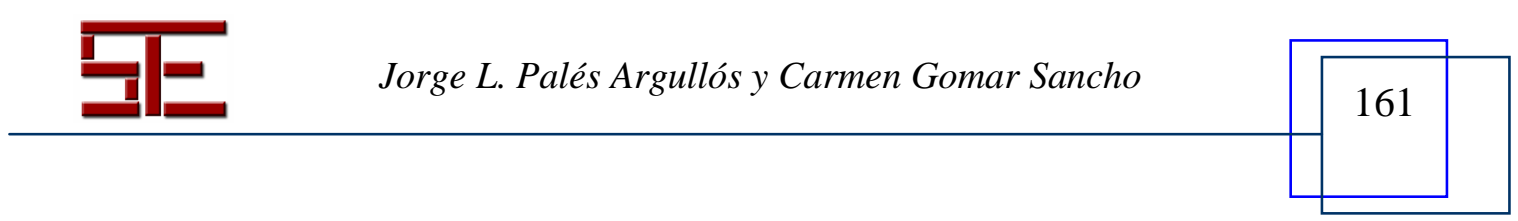




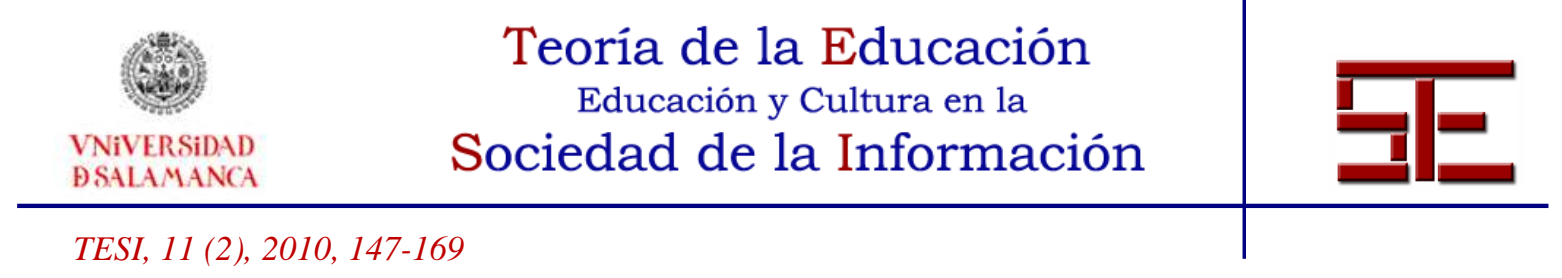

pertenecen a distintas disciplinas de la ciencia (médicos, enfermeras, psicólogos, ingenieros, biólogos, etc.) y otros profesionales del sector o de entidades como los centros de Mainz en Alemania, Herfordshire y Bristol en Gran Bretaña, Porto en Portugal, Herlev en Dinamarca, o los centros de simulación de Santander, Cáceres e Iavante en España.

En nuestro país en junio del año 2009 en Santander, con ocasión de unas jornadas sobre simulación, se dio el primer paso para constituir la Sociedad Española de Simulación en Salud que pretende agrupar a distintas especialidades médicas y quirúrgicas, enfermería y profesionales no sanitarios, como docentes, ingenieros e psicólogos y las principales empresas del sector. con el fin de compartir objetivos y puntos de vista que permitan el desarrollo de estas nuevas metodologías en educación.

\section{7.- REQUISITOS DE UNA BUENA EDUCACIÓN MÉDICA BASADA EN LAS SIMULACIONES}

Para finalizar quisiéramos referirnos brevemente a una serie de requisitos o principios por lo que se ha de regir una buena educación médica basada en las simulaciones, especialmente en el contexto del Grado, basándonos en nuestra experiencia de 4 años al frente del Laboratorio de Habilidades Clínicas de la Facultad de Medicina de la Universidad de Barcelona y que resumimos en tabla 2.

Sin duda alguna, el desarrollo de una buena educación médica basada en las simulaciones requiere inversión en recursos materiales y humanos, pero no menos cierto que dichas premisas son condiciones necesarias pero no son suficientes. Deben ir acompañadas de una serie de condiciones que van a ser indispensables para que esta docencia sea eficaz.

1 La docencia por simulación debe basarse en una estricta planificación de acuerdo con unos objetivos docentes claramente establecidos. Cada enseñanza debe planificarse con un guión que refleje claramente la situación que se va a entrenar, los objetivos que se buscan y las competencias que se van a adquirir. La implementación de estos guiones estará bien sistematizada. Los listados de actividades que deben realizar los participantes servirán para la retroalimentación de la acción de entrenamiento.

2 En el caso del grado, la enseñanza de las habilidades en entornos de este tipo ha de estar integrada perfectamente en el currículum y en relación con la actividad clínica del estudiante. Lo que se enseña debe ser relevante en el contexto. Se debe planificar la enseñanza de las diferentes habilidades de forma integrada con la

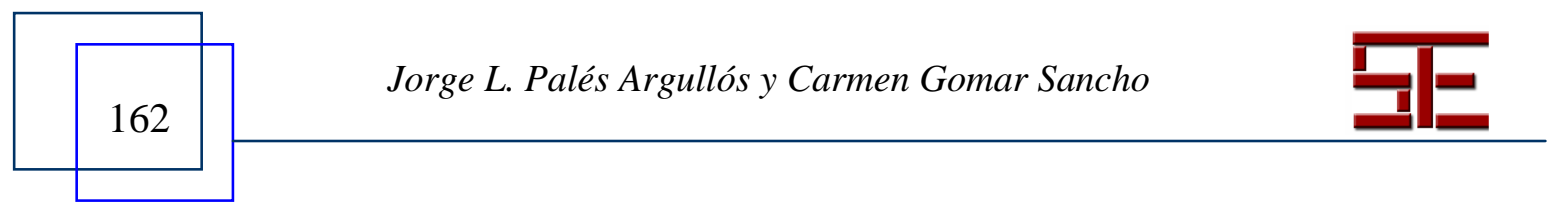




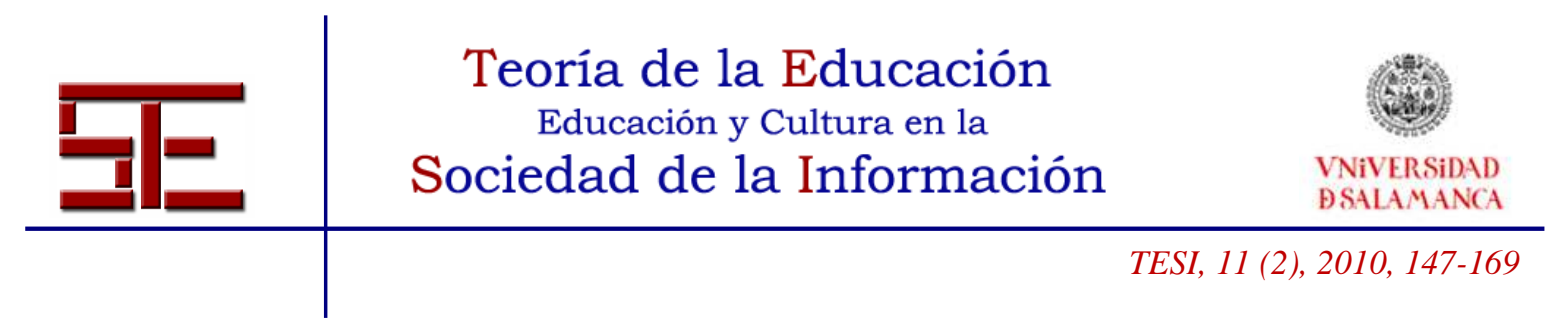

enseñanza teórica y clínica que ha de recibir el alumno y el material que se adquiera debe estar en perfecta consonancia con los objetivos de aprendizaje que se deseen alcanzar.

3 La evaluación es una parte esencial del proceso como en cualquier otra actividad educativa, tanto en su vertiente formativa como sumativa, pero especialmente en la primera. La retroalimentación es una de las partes imprescindibles de la simulación. El pensamiento reflexivo y crítico sobre el entrenamiento realizado debe complementar la retroalimentación, para ir más allá de un acto puramente técnico. La evaluación debe realizarse siempre y para que sea correcta, la simulación ha de tener criterios de validez y reproducibilidad para asegurar que cada grupo entrena las mismas competencias. Este criterio es sobre todo muy importante en la evaluación de tipo sumativo. Los instrumentos de evaluación deben estar bien calibrados conteniendo todas las características claves a evaluar.

4 El entrenamiento basado en la simulación consume tiempo para prepararla y ejecutarla; el papel de profesor desaparece y se sustituye por el de tutor. La preparación de los tutores no es intuitiva, requiere entrenamiento. Las características del tutor en este campo se pueden resumir en: gustarle la docencia, tener conocimiento y recursos docentes, ser accesible, crear un clima positivo, tener una escucha activa, no ser intrusivo, generar preguntas, aprovechar la interacción de los alumnos de un mismo grupo y promover el aprender a aprender.

5 Los laboratorios de habilidades no pueden ser centros aislados del entorno clínico real y se debe ser consciente de sus limitaciones y de las limitaciones de la tecnología. El manejarse correctamente con el simulador no es igual a competencia clínica.

6 Por el elevado costo que representa un laboratorio o centro de este tipo, es necesaria una adecuada conexión con los centros hospitalarios relacionados. Los laboratorios de las facultades y los centros sanitarios deben compartir los recursos tanto materiales como humanos para mejor rentabilizar los mismos y estos deben utilizar las prestaciones de aquellos para la formación continua de sus profesionales.

7 Todo el profesorado y en especial de campos clínicos, deben involucrarse en las actividades de los laboratorios de habilidades y en su planificación y conocer todo lo que en ellos se enseña. Las instituciones deben incluir los claramente la en su política docente y tanto profesores como responsables deben facilitar a sus estudiantes todo el soporte necesario cuando estos desarrollen en la realidad las diferentes habilidades aprendidas en el laboratorio. Los profesores de cada materia

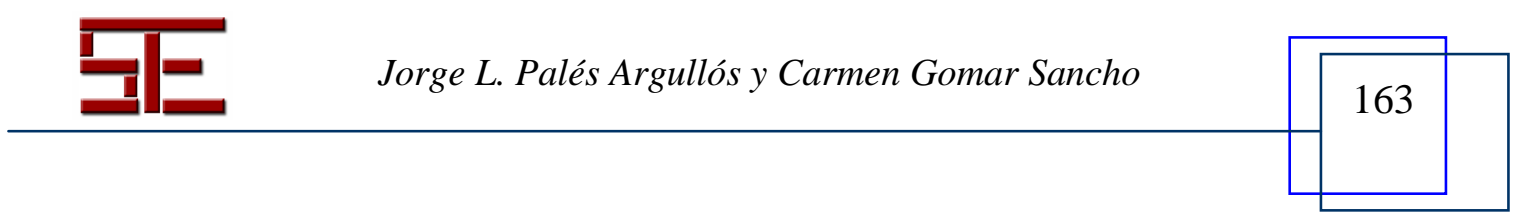




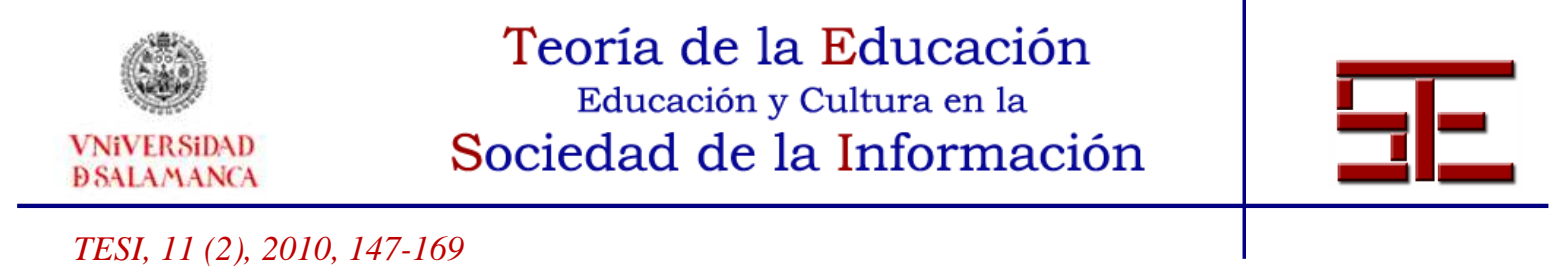

son los responsables de definir los objetivos e instrumentos docentes para la enseñanza por simulación de sus materias.

8 El laboratorio de habilidades debe disponer de personal que asegure su mantenimiento y el de sus recursos y que el profesorado encuentre disponible y sin esfuerzo añadido, el material que precisa. Debe existir personal administrativo que asegure un correcto funcionamiento del mismo y un libre acceso en amplios períodos de tiempo diarios a todos los posibles usuarios y personal técnico especializado en el manejo y mantenimiento de los recursos. La complejidad tecnológica de determinados recursos exige la existencia de este personal para rentabilizar las prestaciones que estos recursos poseen.

9 Los usuarios de los laboratorios de habilidades deben ser conscientes de que aunque trabajan en un entorno de simulación, han de actuar de la misma manera como lo harían en la realidad. El material de simulación no puede considerarse como un mero juguete y en su manejo han de observarse las mismas condiciones de uso y seguridad que en la realidad.

Sin duda alguna en los próximos años asistiremos a un desarrollo cada vez mayor del uso de las simulaciones en educación médica en nuestro país. Debemos ser buenos conocedores de sus características para poder sacar el mejor rendimiento del uso de las mismas en nuestro entorno. Confiamos que este artículo pueda servir de ser ayuda para conseguirlo.

\section{8.- BIBLIOGRAFÍA}

Cantrll, M. (2009). Simulated And Standardized Patients, En Dent, J. Y Harden, R.M. (eds.) A Practical Guide for Medical Teachers, Edinburgh, 224-227 Chpra, V., Engbers, Fhm., Geerts, Mj Et Al. (1994). The Leiden Anaesthesia $\begin{array}{lllll}\text { Simulator. } & \mathrm{Br} & \mathrm{J} & \text { Anaesth } & \text { 287-292. }\end{array}$ Chhristensen, Uj., Andersen, Sf. Jacobsen, J. (1997). The SOPHUS anaesthesia simulator v. 2.0 A Windows 95 control-centre of a full scale simulator. Int J Clin Monit Comput 14: 11-16

Denson, Js., Abrahamson,S. (1969). A computer-controlled patient simulator. JAMA 208 (3) 504-508.

Gaba, D. M., Deanda, A. (1988). A comprehensive-anaesthesia simulation environments: re-creating the operating room for research and training. Anaesthesiology, 69, 387-394

http://www.bmsc.co.uk/sim_database/centres_europe.htm (consultado diciembre 2009) http://www.ccmijesususon.com/ (consultado enero 2010)

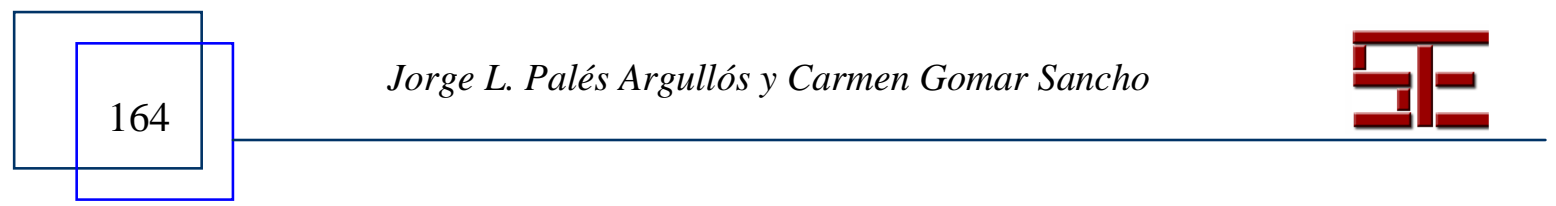




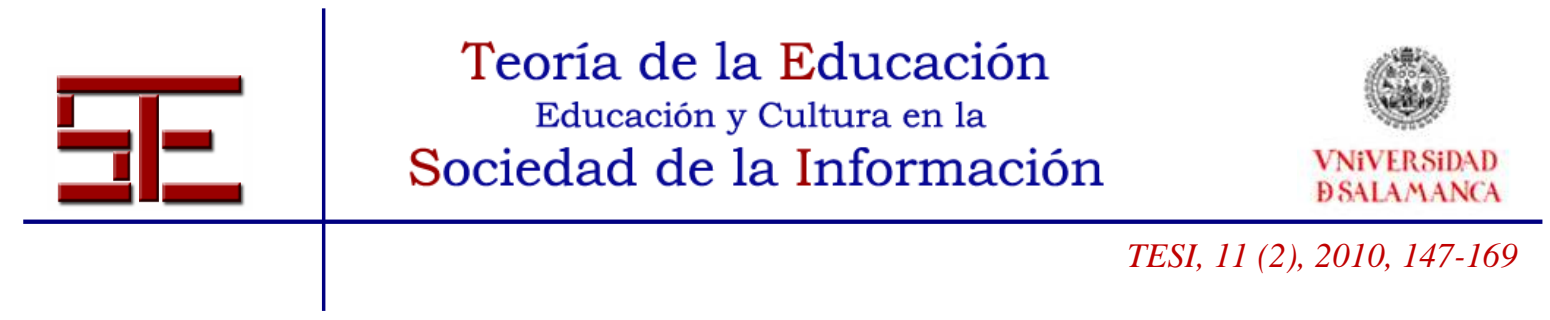

http://www.cesc.com.es/ (consultado enero 2010)

http://www2.iavante.es/es/fundacion (consultado enero 2010)

Horley, R. Simulation And Skill Centre Design En Riley, R.H. (ed.) (2008). Manual of simulation in Healthcare, Oxford University Press, 3-10.

Kohn, L.T., Corrigan J.M., And Donaldson, M.S. (2000). To err is human: building a safer health system. National Academy Press, Washington, D.C.

Link Ea. US Patent 1,825, 462. Filed 1930

Mazarro, A., Palés, J. Gomar, C. (2009). Implementación de un laboratorio de habilidades centralizado en la Facultad de Medicina de la Universidad de Barcelona. Cuatro años de experiencia. Educación Médica 12(4) en prensa.

Rettedal, A, Freyer, S., Kleppa, R. Larsen, P. (1996). PatSim- simulator for practicing anesthesia and intensive care. Int J Clin Monit Comput 14, 11-16

The Israel Centre For Medical Simulation. http://www.msr.org.il (consultado en enero 2010).

Vázquez-Mata, G, Modelos, estrategias y tendencias en España de la simulación en Medicina. Educación Médica 10(3): 147-148

Vázquez-Mata, G., Guillamer Lloveras, A. (2009). El entrenamiento basado en la simulación como innovación imprescindible en la formación médica

Educación Médica 12(3), 149-145

Ziv, A., Wolpe, P., Small, S., Glick, S. (2003). Simulation-based medical education- an ethical imperative. Academic Medicine, 78, 783-788.

Ziv, A. (2007). Simulation-Based Medical Education -From Vision to Reality. Educación Médica. 10(3): 147-148

Ziv, A, Berkenstad, H (2008). La educación médica basada en simulaciones. JANO, $170142-45$

Ziv, A. (2009). Simulators and simulation-based medical education, en Dent, J. y Harden, R.M. (eds.) A Practical Guide for Medical Teachers, Edinburgh, 217-222.

Simuladores de baja tecnología.

Simuladores sencillos mecánicos, de plástico o sintéticos

Cadáveres humanos

Animales

Pacientes simulados/estandardizados

Simuladores de alta tecnología

Simulaciones por ordenador o mediante "pantalla" (screen simulation).

Simuladores informáticos de gran fidelidad con recursos audiovisuales y táctiles

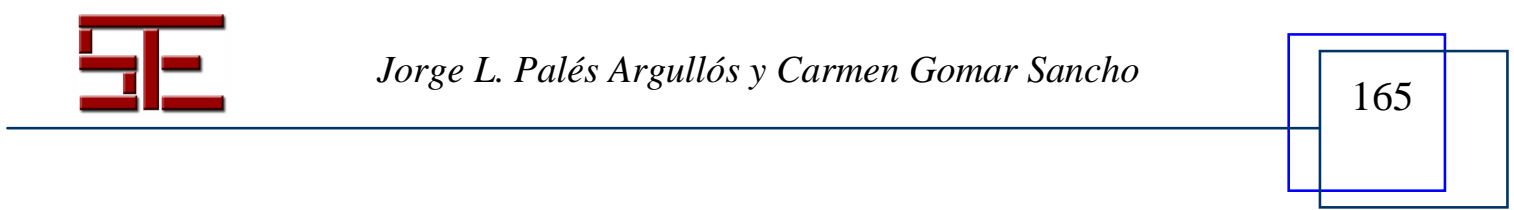




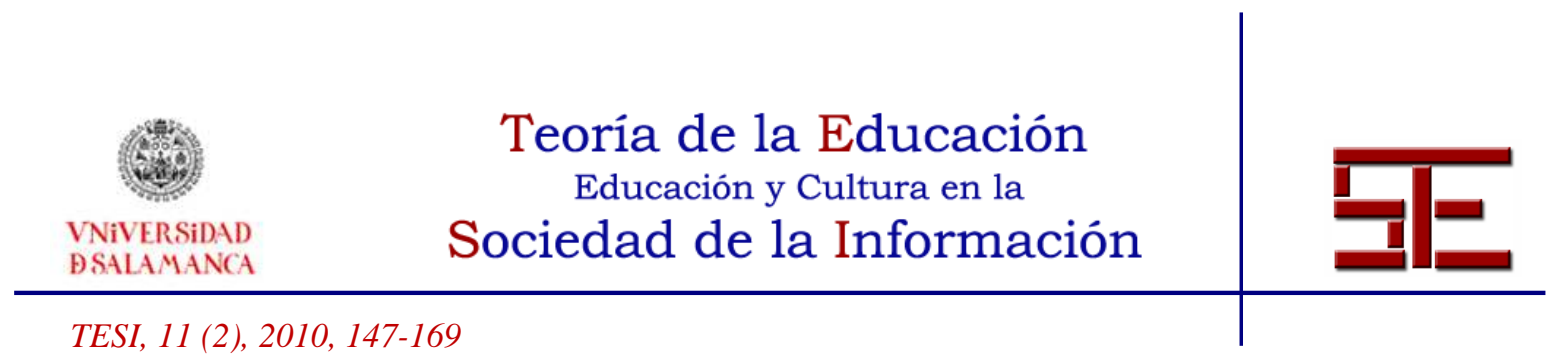

Simuladores de paciente completo interactivo realístico y de alta tecnología.

Tabla 1: Recursos disponibles para simulación

1. Planificación estricta de acuerdo con unos objetivos docentes claramente establecidos.

2. Integración en el curriculum y relación con la actividad clínica del estudiante.

3. Necesidad del feed-back como elemento esencial de la simulación.

4. Tiempo suficiente para preparar y ejecutar la simulación

5. Necesidad de formación y dedicación del profesor/tutor

6. Conciencia de las limitaciones de la simulación y necesidad de tener en cuenta siempre el entorno clínico real

7. Adecuada conexión con los centros hospitalarios relacionados y compartición de los recursos

8. Implicación de todo el profesorado y de la institución

9. Disponibilidad de personal técnico y de mantenimiento.

10. Los usuarios deben actuar del mismo modo como lo harían en la realidad

Tabla 2: Requisitos para la educación médica basada en la simulación en facultades de medicina

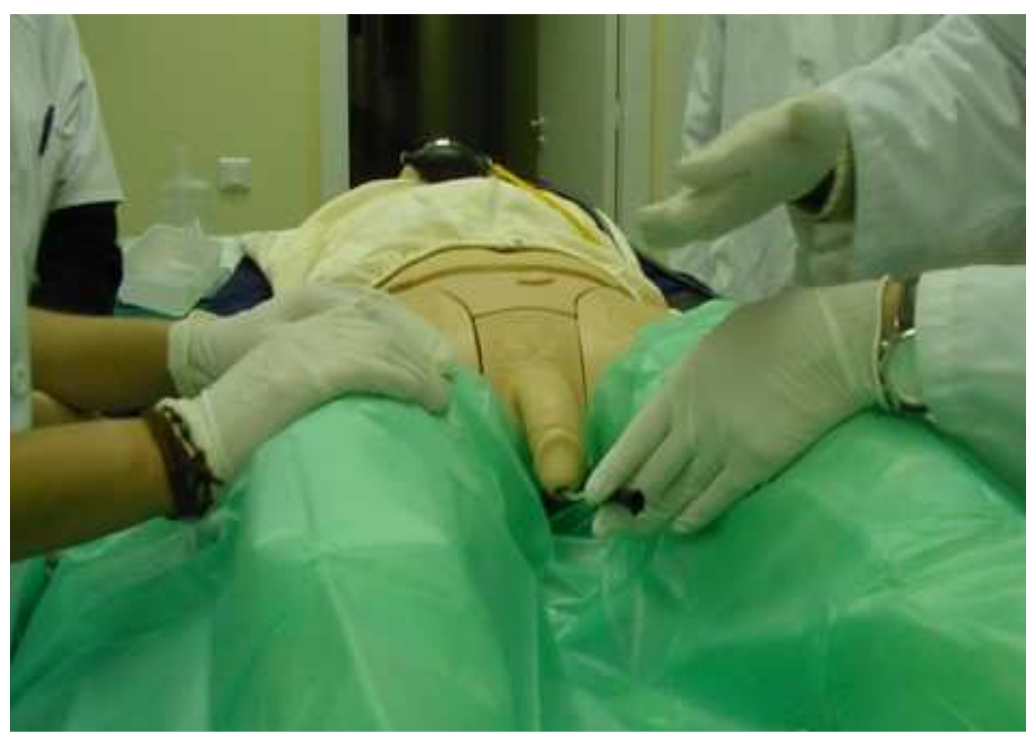

Figura 1: Práctica del sondaje uretral mediante simulación

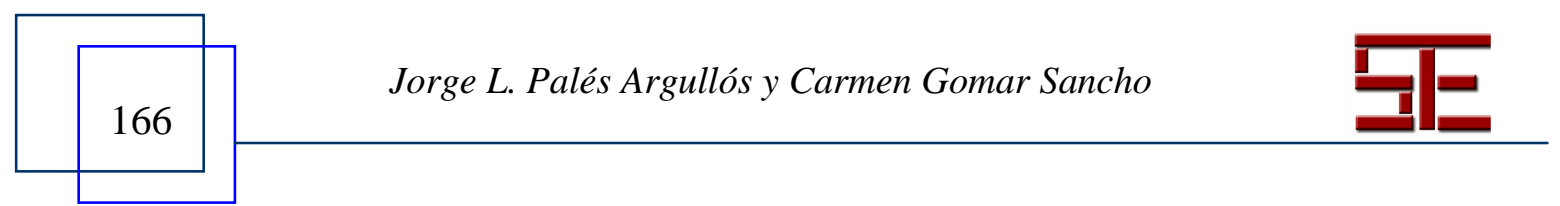




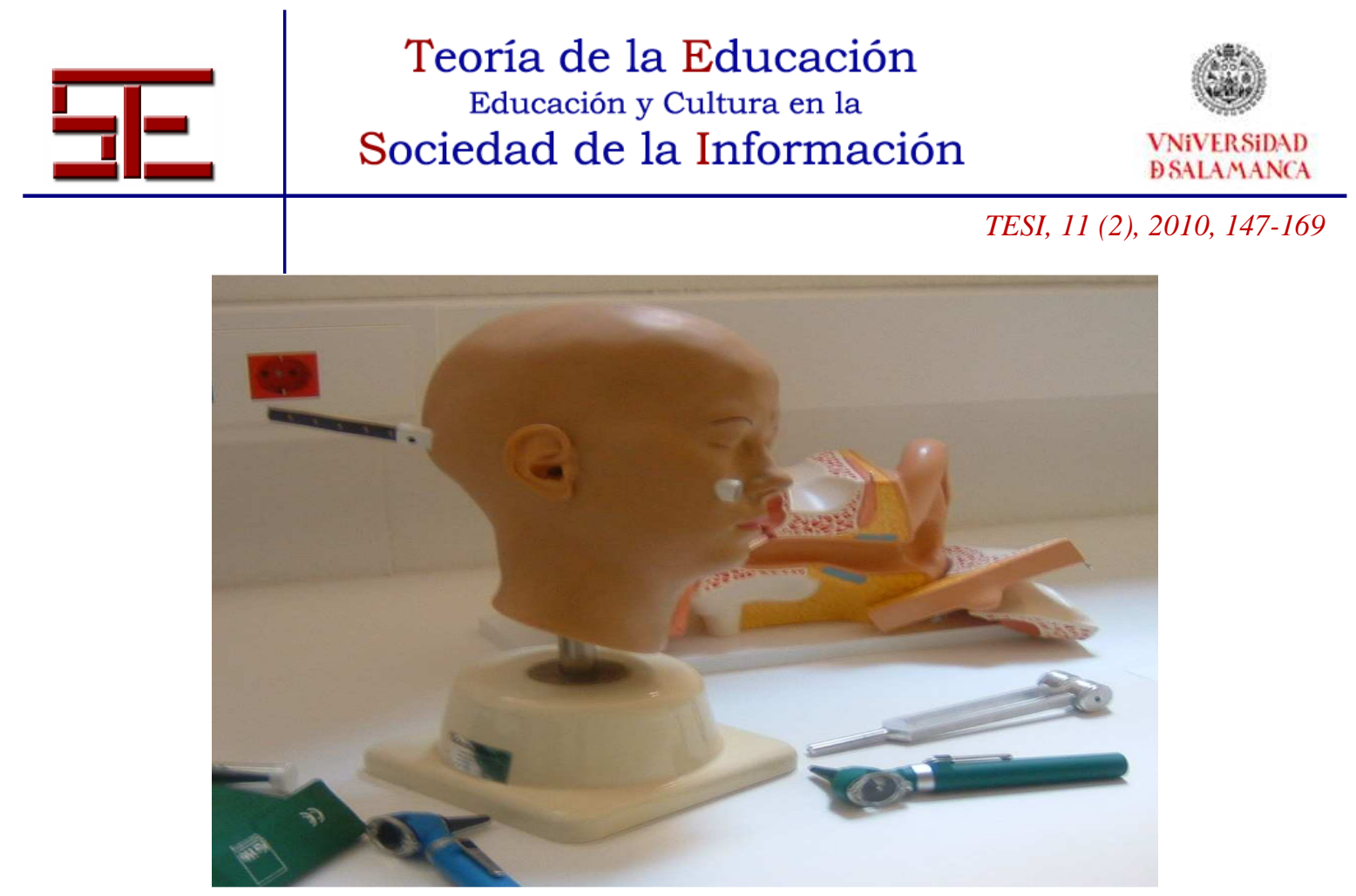

Figura 2: Simulador para exploración otorrinolaringológica

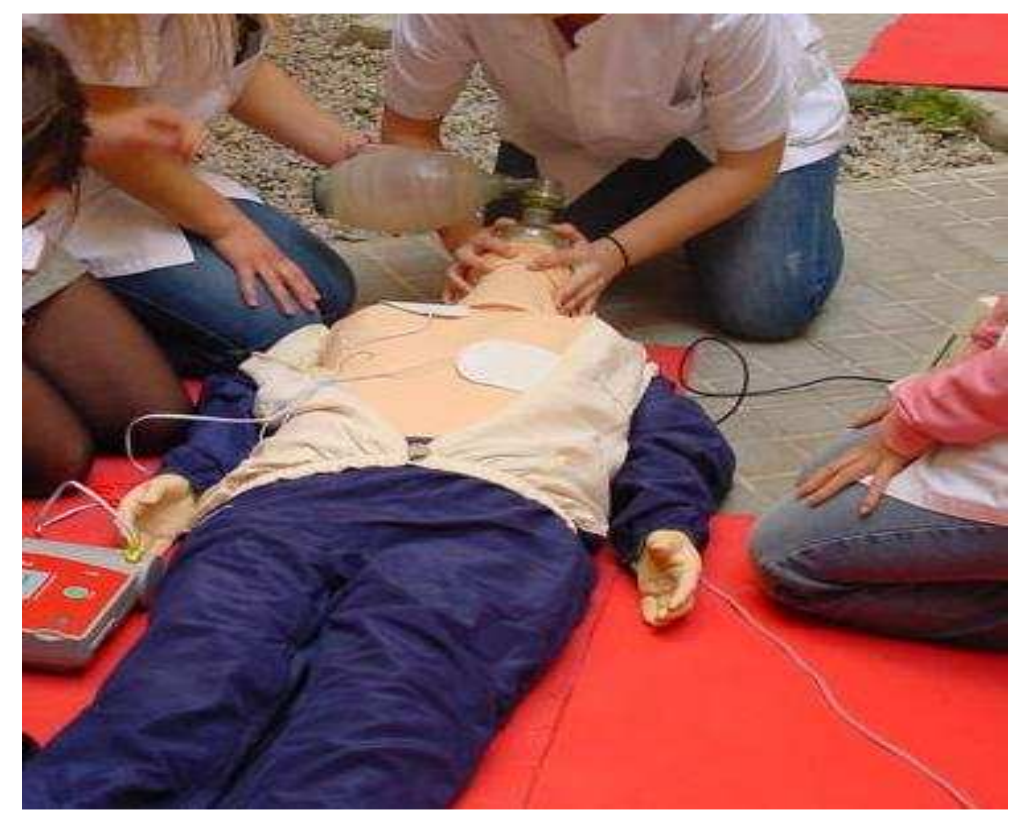

Figura 3: Prácticas de Resucitación cardiopulmonar mediante simulación

Jorge L. Palés Argullós y Carmen Gomar Sancho 


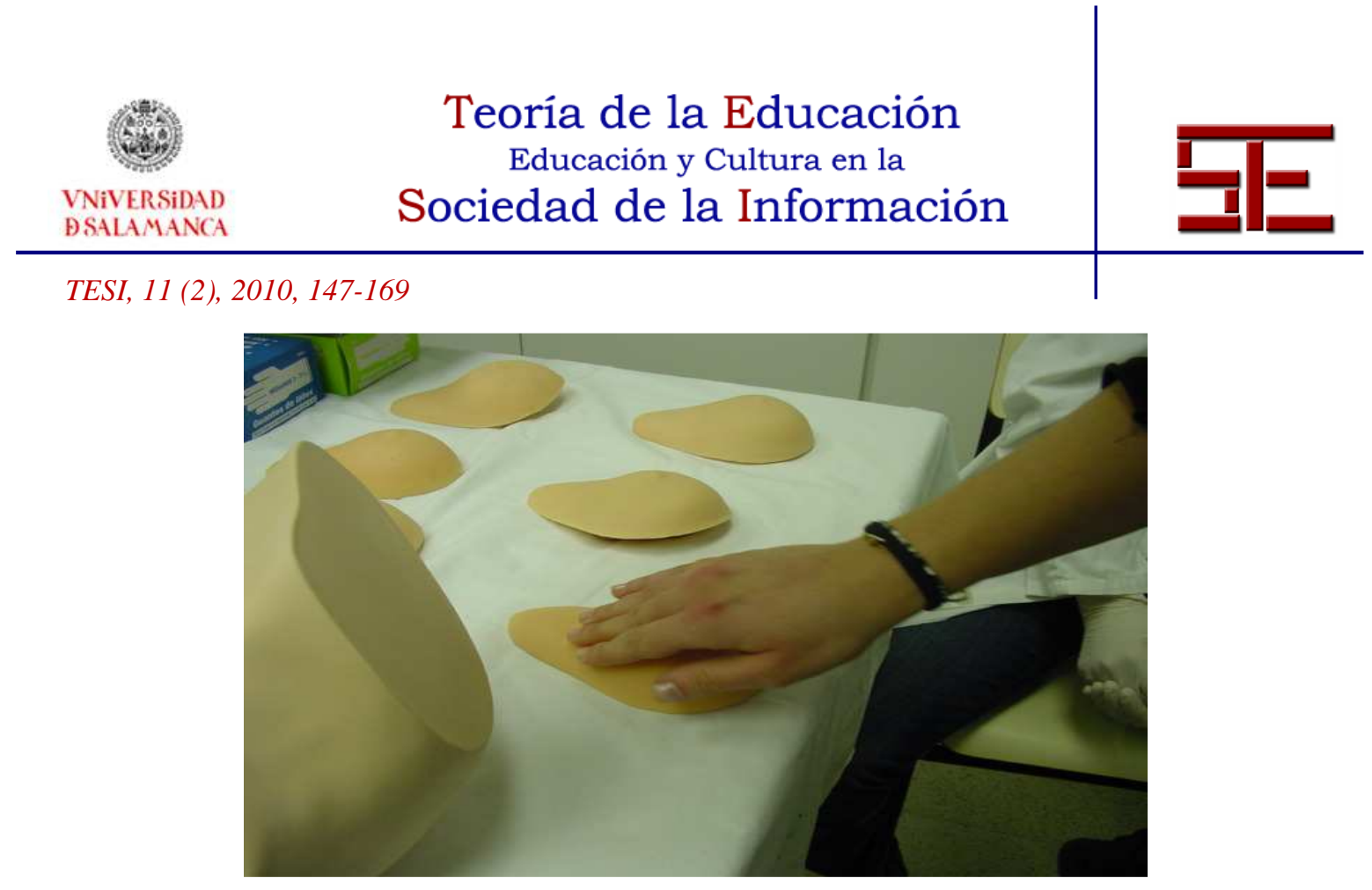

Figura 4: Simulador para exploración mamaria

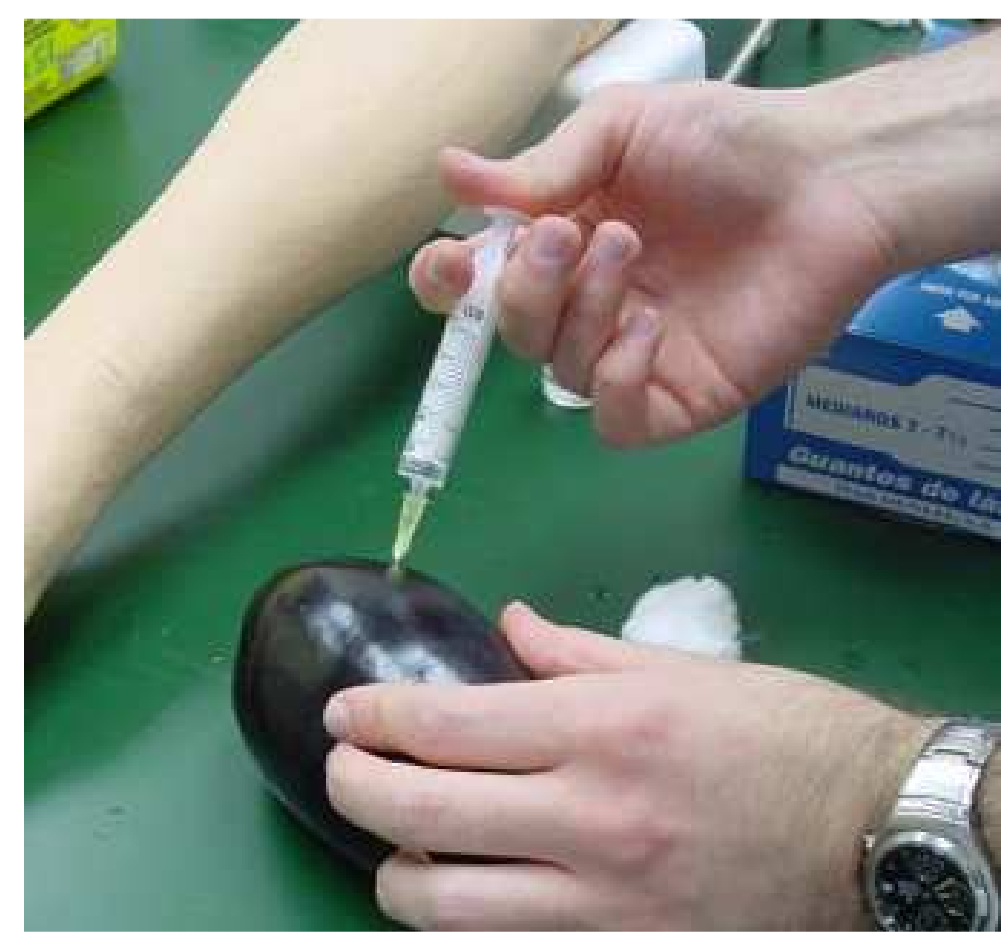

Figura 5: Práctica de punción intramuscular usando una simulación sencilla (una berenjena)”

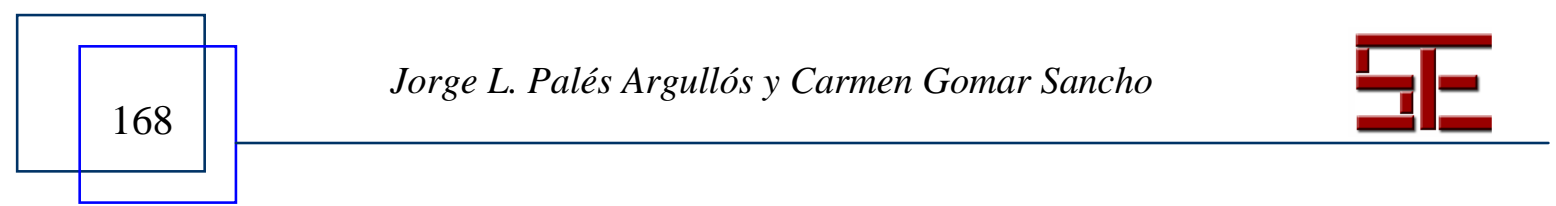




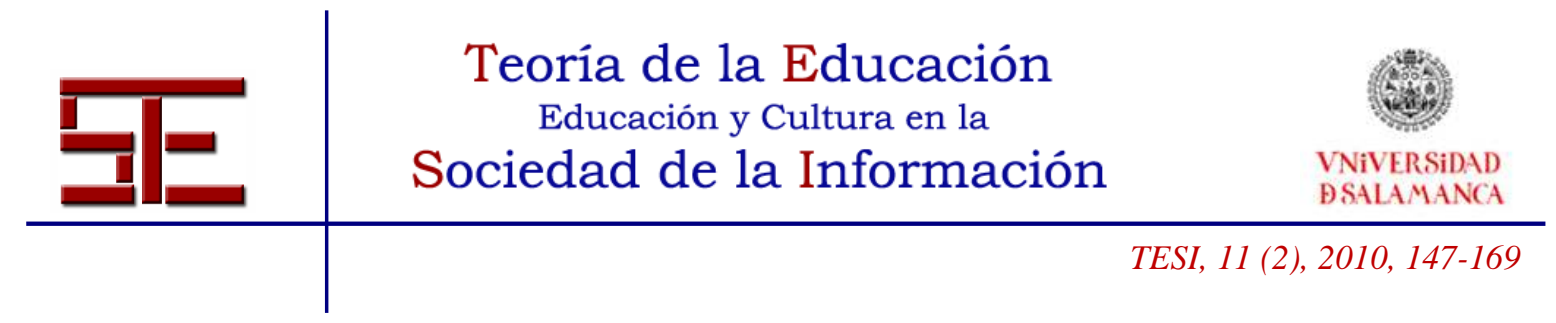

Para citar el presente artículo puede utilizar la siguiente referencia:

Palés Argullós, J.L. y Gomar Sancho, C. (2010): El uso de las simulaciones en Educación Médica, en Juanes Méndez, J. A. (Coord.) Avances tecnológicos digitales en metodologías de innovación docente en el campo de las Ciencias de la Salud en España. Revista Teoría de la Educación: Educación y Cultura en la Sociedad de la Información. Vol. 11, n 2. Universidad de Salamanca, pp. 147-169 [Fecha de consulta: dd/mm/aaaa].

http://campus.usal.es/ revistas_trabajo/index.php/revistatesi/article/view/7075/7108

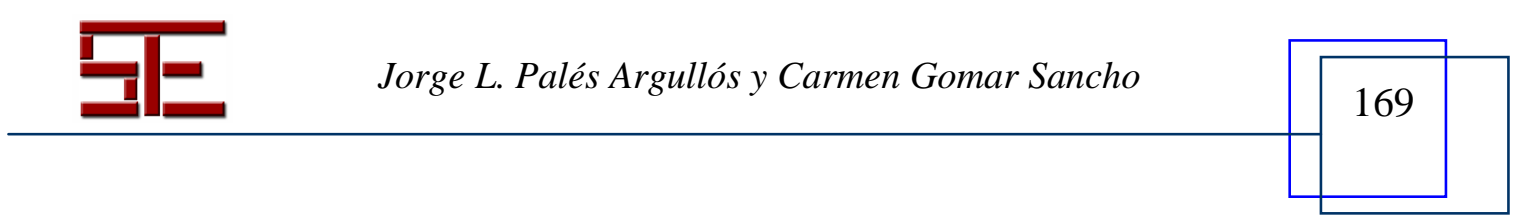

